\title{
TRIGONOMETRY IN EXTENDED HYPERBOLIC SPACE AND EXTENDED DE SITTER SPACE
}

\author{
YunHI CHO
}

\begin{abstract}
We study the hyperbolic cosine and sine laws in the extended hyperbolic space which contains hyperbolic space as a subset and is an analytic continuation of the hyperbolic space. And we also study the spherical cosine and sine laws in the extended de Sitter space which contains de Sitter space $S_{1}^{n}$ as a subset and is also an analytic continuation of de Sitter space. In fact, the extended hyperbolic space and extended de Sitter space are the same space only differ by -1 multiple in the metric. Hence these two extended spaces clearly show and apparently explain that why many corresponding formulas in hyperbolic and spherical space are very similar each other. From these extended trigonometry laws, we can give a coherent and geometrically simple explanation for the various relations between the lengths and angles of hyperbolic polygons, and relations on de Sitter polygons which lie on $S_{1}^{2}$, and tangent laws for various polyhedra.
\end{abstract}

\section{Introduction}

There are well known hyperbolic cosine and sine laws for triangles in the hyperbolic space $\mathbb{H}^{n}$. If we consider Kleinian model which contains the hyperbolic space as an open ball, we can think about more general triangle which lies outside the hyperbolic space or intersects the ideal boundary $\partial \mathbb{H}^{n}$. Then there is a difficulty in geometric interpretation of such general type triangle or other geometric objects. However the extended hyperbolic space which is an analytic continuation of the hyperbolic space can give a playground for such general geometric objects. Similarly extended de Sitter space is obtained from de Sitter space $S_{1}^{n}$ and shows the phenomena of the spherical geometry $\mathbb{S}^{n}$, just like the extended hyperbolic space shows that of the hyperbolic geometry $\mathbb{H}^{n}$.

In Section 2, we discuss what the extended model is and how it can be constructed. The extended hyperbolic space which contains hyperbolic space as a subset looks like the unit sphere $\mathbb{S}^{n}$ topologically. More detailed explanations about the extended space are given in [2].

Received September 4, 2008.

2000 Mathematics Subject Classification. 51M10, 51M25, 53B30.

Key words and phrases. hyperbolic space, volume, analytic continuation.

This work was supported by the Research Fund 2006 of University of Seoul. 
In Section 3, we explain how to define the notions of length and angle on the extended space. In order to understand the extended space more precisely, we should use $\epsilon$-approximation technique. However here we only consider simple geometric objects such as length and angle, and we need not deeper theory of the model. Here the length and angle must take complex values in general. This kind of complex valued angle was introduced by Dzan ([5], [6]). He also constructed natural flat Lorentzian geometry on $\mathbb{R}^{n, 1}$ that looks like Euclidean geometry on $\mathbb{R}^{n+1}$, then many formulas on $\mathbb{R}^{n, 1}$ and $\mathbb{R}^{n+1}$ exactly coincide each other. Schlenker [12] also defined complex valued distance and angle on Kleinian model using cross ratio. Our approach to distance and angle on the extended space is more geometrically motivated and simple, and turned out to be the same as Dzan and Schlenker's.

In Section 4, we prove the generalized hyperbolic (resp. spherical) cosine and sine laws for the extended hyperbolic (resp. extended de Sitter) space, those laws have exactly the same representation (see Theorems 4.13 and 4.15 ) of the original hyperbolic space $\mathbb{H}^{n}$ (resp. spherical space $\mathbb{S}^{n}$ ). Note that most of the proof and its difficulty for the generalized cosine and dual cosine laws come from the sign $( \pm)$ determining process. These generalized cosine and sine laws explain and easily deduce the well-known relations (see Fenchel's book [8] or [11] or [13]) about the lengths and angles of hyperbolic polygons in a simple unified way, for example, Lambert quadrilateral, pentagon, rectangular hexagon, and so on. Furthermore we can also obtain the similar relations between the lengths and angles of de Sitter polygons on the pseudo-sphere $(=$ Lorentz space of constant curvature 1) $S_{1}^{2}$. As an three dimensional application, we show a unified method about the tangent laws on some simple hyperbolic or spherical polyhedra.

Lastly we remark some problems at the end of the paper which seem to be important phenomena between the hyperbolic space $\mathbb{H}^{n}$ and the spherical space $\mathbb{S}^{n}$.

Acknowledgement. The author would like to thank to Hyuk Kim and Hyounggyu Choi. They gave some helpful comments for this paper.

\section{Extended hyperbolic space and extended de Sitter space}

Our main concern is the unified trigonometry on the extended space, so we should know what the extended space is and why we need to know the trigonometry on the model. For the answer of the first question, the model is well explained in [2] and reader can easily understand the extended hyperbolic model itself and the importance of the model. However we will introduce some necessary parts of the theory in the following for convenience. And the second question will be considered in Section 4.

To define and explain the extended model, let's start with the hyperboloid model of hyperbolic space. Let $\mathbb{R}^{n, 1}$ denote the real vector space $\mathbb{R}^{n+1}$ equipped 
with the bilinear form of signature $(n, 1)$,

$$
\langle x, y\rangle=-x_{0} y_{0}+x_{1} y_{1}+\cdots+x_{n} y_{n}
$$

for all $x=\left(x_{0}, x_{1}, \ldots, x_{n}\right), y=\left(y_{0}, y_{1}, \ldots, y_{n}\right)$. Then the hyperbolic spaces $H_{+}^{n}$ and $H_{-}^{n}$, pseudo-sphere $S_{1}^{n}$ and light cone $L^{n}$ are defined by

$$
\begin{aligned}
H_{+}^{n} & :=\left\{x \in \mathbb{R}^{n, 1} \mid\langle x, x\rangle=-1, \quad x_{0}>0\right\}, \\
H_{-}^{n} & :=\left\{x \in \mathbb{R}^{n, 1} \mid\langle x, x\rangle=-1, \quad x_{0}<0\right\}, \\
S_{1}^{n} & :=\left\{x \in \mathbb{R}^{n, 1} \mid\langle x, x\rangle=1\right\}, \\
L^{n} & :=\left\{x \in \mathbb{R}^{n, 1} \mid\langle x, x\rangle=0\right\} .
\end{aligned}
$$

We already know that $H_{ \pm}^{n}$ has the induced Riemannian manifold structure which has a constant sectional curvature -1 , and that $S_{1}^{n}$ becomes a Lorentzian manifold (or semi-Riemannian of signature $(-,+, \ldots,+)$ ) which has a constant sectional curvature 1, also called as de Sitter space (see [10]). Now we consider the Kleinian projective model. By the radial projection $\pi_{1}$ with respect to the origin from $H_{+}^{n}$ onto $\{1\} \times \mathbb{R}^{n}$, we obtain the induced Riemannian metric on the ball in $\{1\} \times \mathbb{R}^{n}$ as follows $([1],[11])$,

$$
d s_{K}^{2}=\left(\frac{\Sigma x_{i} d x_{i}}{1-|x|^{2}}\right)^{2}+\frac{\Sigma d x_{i}^{2}}{1-|x|^{2}} .
$$

If we extend this metric beyond the unit ball using the same formula, this metric induces a semi-Riemannian structure outside the unit ball in $\{1\} \times \mathbb{R}^{n}$. In fact, we compare this metric with the one induced from the Lorentzian space $S_{1}^{n} \cap\left\{x=\left(x_{0}, x_{1}, \ldots, x_{n}\right) \mid x_{0}>0\right\}$, by the radial projection into $\{1\} \times \mathbb{R}^{n}$, then they differ only by sign -1 . This sign change of the metric implies the sign change of the sectional curvature from +1 to -1 , which, of course, the curvature of the metric $d s_{K}^{2}$. In this way, we obtain an extended Kleinian model with a singular metric $d s_{K}^{2}$ defined on $\{1\} \times \mathbb{R}^{n}$, and this extended hyperbolic space $\left(\{1\} \times \mathbb{R}^{n}, d s_{K}^{2}\right)$ will be denoted by $K^{n}$.

In this paper, it is more convenient to consider the Euclidean unit sphere in $\mathbb{R}^{n+1}$ with the induced metric coming from $H_{ \pm}^{n}$ and $-S_{1}^{n}\left(S_{1}^{n}\right.$ with $-1 \times$ its metric) via radial projection, and denote this model by $\mathbb{S}_{H}^{n}$. This hyperbolic sphere model $\mathbb{S}_{H}^{n}$ (another model of the extended hyperbolic space) on the Euclidean sphere $\left\{x=\left(x_{0}, x_{1}, \ldots, x_{n}\right) \mid x_{0}^{2}+x_{1}^{2}+\cdots+x_{n}^{2}=1\right\}$ has three parts: Two radial images of $H_{ \pm}^{n}$, called the hyperbolic part, as two open disks on upper and lower hemisphere and the radial image of $S_{1}^{n}$, called the Lorentzian part, and these all three parts have constant sectional curvature -1 .

We can study the geometry of $\mathbb{S}_{H}^{n}$ as an analytic continuation of the hyperbolic space $\mathbb{H}^{n}$. First let's define the volume of a set on the hyperbolic sphere. We denote $d V_{K}$ and $d V_{S}$ for the volume forms on $K^{n}$ and $\mathbb{S}_{H}^{n}$ respectively. From the metric of the extended Kleinian model, we have the following volume 
form $d V_{K}$ (see $\S 6.1$ of [11]).

$$
\begin{aligned}
d V_{K} & =\left(\operatorname{det}\left(g_{i j}\right)\right)^{\frac{1}{2}} d x_{1} \wedge \cdots \wedge d x_{n}, \\
& =\frac{d x_{1} \wedge \cdots \wedge d x_{n}}{\left(1-|x|^{2}\right)^{\frac{n+1}{2}}} .
\end{aligned}
$$
by

For any set $U$ on $\mathbb{S}_{H}^{n} \cap\left\{x \in \mathbb{R}^{n, 1} \mid x_{0}>0\right\}$, we can evaluate the volume of $U$

$$
\begin{aligned}
\operatorname{vol}(U) & =\int_{U} d V_{S} \\
& =\int_{\pi^{-1}(U)} d V_{K} \quad\left(\text { where } \pi \text { is a radial projection: } K^{n} \rightarrow \mathbb{S}_{H}^{n} \cdot\right) \\
& =\int_{\pi^{-1}(U)} \frac{d x_{1} \wedge \cdots \wedge d x_{n}}{\left(1-|x|^{2}\right)^{\frac{n+1}{2}}} \\
& =\int_{G^{-1}\left(\pi^{-1}(U)\right)} \frac{r^{n-1}}{\left(1-r^{2}\right)^{\frac{n+1}{2}}} d r \wedge d \theta
\end{aligned}
$$

where $G:(r, \theta) \mapsto\left(x_{1}, \ldots, x_{n}\right)$ is the polar coordinates and $d \theta$ is the volume form of the Euclidean sphere $\mathbb{S}^{n-1}$.

If $F(r)=\int_{G^{-1}(U) \cap S^{n-1}(r)} d \theta$ is an analytic function of $r$, then the above integral becomes a 1-dimensional integral as follows.

$$
\int_{G^{-1}(U)} \frac{r^{n-1}}{\left(1-r^{2}\right)^{\frac{n+1}{2}}} d r d \theta=\int_{a}^{b} \frac{r^{n-1} F(r)}{\left(1-r^{2}\right)^{\frac{n+1}{2}}} d r .
$$

In general this integral does not make sense and the most natural thing we can do instead is to define $\operatorname{vol}(U)$ as the following contour integral:

\section{Convention 2.1 .}

$$
\operatorname{vol}_{H}(U):=\int_{\gamma} \frac{r^{n-1} F(r)}{\left(1-r^{2}\right)^{\frac{n+1}{2}}} d r
$$

where $\gamma$ is a contour from $a$ to $b$ for $a<1<b$ as depicted below. Here we will fix its contour type as clockwise around $z=1$ once and for all throughout the paper.

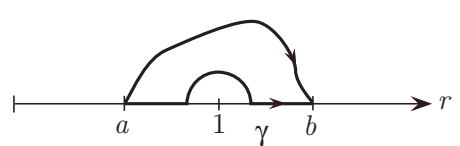

Fig. 1 
Therefore we can compute the length of line segment on $\mathbb{S}_{H}^{1}$ by using the line integral (2). It is easy to see that

$$
d_{H}(0, b):=\int_{0, \gamma}^{b} \frac{d r}{1-r^{2}}=\left\{\begin{array}{lr}
\frac{1}{2} \log \frac{1+b}{1-b}, & 0 \leq b<1, \\
\frac{1}{2} \log \frac{b+1}{b-1}+\frac{\pi}{2} i, & 1<b,
\end{array}\right.
$$

where $d_{H}$ denotes 1-dimensional extended hyperbolic length of the line segment $[0, b]$ in $K^{1}$ in the sense of $(2)$.

If $F(r)$ is an analytic function around $r=1$, then it is easily shown (see Proposition 2.1 of [2]) that

$\lim _{\epsilon \rightarrow 0} \int_{U} d V_{K, \epsilon}:=\lim _{\epsilon \rightarrow 0} \int_{U} \frac{d_{\epsilon} r^{n-1}}{\left(d_{\epsilon}^{2}-r^{2}\right)^{\frac{n+1}{2}}} d r d \theta=\operatorname{vol}_{H}(U)$, where $d_{\epsilon}=1-\epsilon i(\epsilon>0)$.

We called the above limit type approach $\epsilon$-approximation technique which is more useful in the theoretical consideration. If we choose $d_{\epsilon}=1+\epsilon i$ instead, then $\lim _{\epsilon \rightarrow 0} \int_{U} d V_{K, \epsilon}$ will give a different value and a slightly different geometry. That is exactly corresponding to a contour integration with a counterclockwise around $z=1$, i.e., going around at $z=1$ through lower half plane.

To determine the various geometric quantities which are to be obtained as integrations on $\mathbb{S}_{H}^{n}$, the norms of vectors are essential. From the sign change of the metric on the pseudo-sphere $S_{1}^{n}$, the norms of tangent vectors on the Lorentzian part are calculated by

$$
\left\|x_{p}\right\|^{2}=-\left(-x_{0}^{2}+x_{1}^{2}+\cdots+x_{n}^{2}\right),
$$

and we should determine the sign of $\left\|x_{p}\right\|$ among \pm 1 and $\pm i$. We can find suitable consistent sign choices for the norms of various tangent vectors on $\mathbb{S}_{H}^{n}$. We list the signs of the tangent vectors in Convention 2.2 below and Fig. 2 . See [2] for the explanation for these choices of the signs.

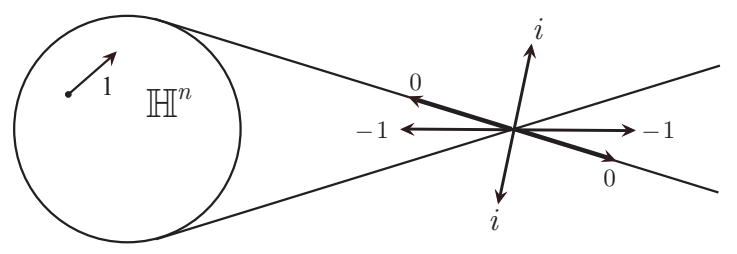

Fig . 2

Convention 2.2. A tangent vector on the hyperbolic part on $\mathbb{S}_{H}^{n}$ has a positive real norm, and a tangent vector on the Lorentzian part on $\mathbb{S}_{H}^{n}$ has a negative real, zero, or positive pure imaginary norm depending on whether it is timelike, lightlike, or spacelike respectively. 
Now let's think about another analytic continuation of the pseudo-sphere $S_{1}^{n}$. Basically the induced metric from $S_{1}^{n} \cap\left\{x \mid x_{0}>0\right\}$ into $\{1\} \times \mathbb{R}^{n}$ by the radial projection differs by -1 from Kleinian metric $d s_{K}^{2}$, and we can extend this metric to the inside of the unit ball. Note that we always fix the sign of the norm of the tangent vector on $\mathbb{S}^{n-1}=S_{1}^{n} \cap\left\{x \mid x_{0}=0\right\}$ as +1 as usual. Hence we will assume the sign of the norm of the spacelike vector on $\mathbb{S}_{S}^{n}$ (see below) as +1 . Here we denote the space and metric as $-K^{n}$ and $d s_{-K}^{2}$ respectively. Then by similar arguments we can consider the unit sphere in $\mathbb{R}^{n+1}$ with induced metric coming from the metric $d s_{-K}^{2}$ by the radial projection, and we call this model as a spherical sphere model, which is one model of the extended de Sitter space, and is denoted by $\mathbb{S}_{S}^{n}$. Also we denote $d V_{-K}$ and $d V_{-S}$ as the volume forms on $-K^{n}$ and $\mathbb{S}_{S}^{n}$ respectively, where $d V_{-K}=(-1)^{\frac{n}{2}} d V_{K}$ and $d V_{-S}=(-1)^{\frac{n}{2}} d V_{S}$. Now we have to fix the exact value of $(-1)^{\frac{n}{2}}$ between $i^{n}$ and $(-i)^{n}$. By comparison of the norm of spacelike tangent vector at a Lorentzian point on $\mathbb{S}_{H}^{n}$ and $\mathbb{S}_{S}^{n},(-i)^{n}$ becomes a reasonable choice between $i^{n}$ and $(-i)^{n}$.

Convention 2.3. For any domain $U$ on $\mathbb{S}_{S}^{n}$, we evaluate the $n$-dimensional volume of $U$ by

$$
\operatorname{vol}_{S}(U)=\int_{U} d V_{-S}=(-i)^{n} \int_{U} d V_{S}=(-i)^{n} \operatorname{vol}_{H}(U) .
$$

From the similar chasing of volume form on $\mathbb{S}_{S}^{1}, \mathbb{S}_{S}^{2}$ and considering of the equator of pseudo-sphere $S_{1}^{n}$, i.e., the Euclidean sphere $\mathbb{S}^{n-1}$, we naturally conclude the following convention.

Convention 2.4. A tangent vector on the hyperbolic part on $\mathbb{S}_{S}^{n}$ has a negative pure imaginary norm, and a tangent vector on the Lorentzian part on $\mathbb{S}_{S}^{n}$ has a positive pure imaginary, zero, or positive real norm depending on whether it is timelike, lightlike, or spacelike respectively (see Fig. 3).

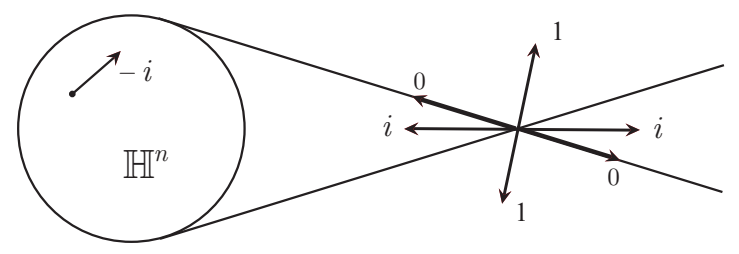

Fig. 3

We can see one of the similarities between $\mathbb{S}_{H}^{n}, \mathbb{S}_{S}^{n}$ and $\mathbb{S}^{n}$ in the following theorem (see [2] and Convention 2.3 for a proof).

Theorem 2.5. $\operatorname{vol}_{H}\left(\mathbb{S}_{H}^{n}\right)=i^{n} \cdot \operatorname{vol}\left(\mathbb{S}^{n}\right)$ and $\operatorname{vol}_{S}\left(\mathbb{S}_{S}^{n}\right)=\operatorname{vol}\left(\mathbb{S}^{n}\right)$. 
If we change the contour type of the integral (2), we have different relation between $\operatorname{vol}_{H}\left(\mathbb{S}_{H}^{n}\right)$ and $\operatorname{vol}\left(\mathbb{S}^{n}\right)$. Also for the various different kinds of contour types, the conventions about $\mathbb{S}_{S}^{n}$ should be changed and the relations between $\operatorname{vol}_{S}\left(\mathbb{S}^{n}\right)$ and $\operatorname{vol}\left(\mathbb{S}^{n}\right)$ have similar modifications as the hyperbolic ones too. If the contour is counterclockwise, then we have $\operatorname{vol}_{H}\left(\mathbb{S}_{H}^{n}\right)=(-i)^{n}$ $\operatorname{vol}\left(\mathbb{S}^{n}\right)$ (by slight change of the proof of Theorem 2.3 in [2]) and $\operatorname{vol}_{S}(U)=$ $i^{n} \operatorname{vol}_{H}(U)$ (counterclockwise version of Convention 2.3). Hence we also get $\operatorname{vol}_{S}\left(\mathbb{S}_{S}^{n}\right)=\operatorname{vol}\left(\mathbb{S}^{n}\right)$.

For various kinds of contour types, we easily deduce the following four formulas,

$$
\begin{aligned}
\operatorname{vol}_{H}\left(\mathbb{S}_{H}^{2 k-1}\right) & \equiv i^{2 k-1} \operatorname{vol}\left(\mathbb{S}^{2 k-1}\right) \quad\left(\bmod 2 i^{2 k-1} \operatorname{vol}\left(\mathbb{S}^{2 k-1}\right)\right) \\
\operatorname{vol}_{H}\left(\mathbb{S}_{H}^{2 k}\right) & =i^{2 k} \operatorname{vol}\left(\mathbb{S}^{2 k}\right) \\
\operatorname{vol}_{S}\left(\mathbb{S}_{S}^{2 k-1}\right) & \equiv \operatorname{vol}\left(\mathbb{S}^{2 k-1}\right) \quad\left(\bmod 2 \operatorname{vol}\left(\mathbb{S}^{2 k-1}\right)\right) \\
\operatorname{vol}_{S}\left(\mathbb{S}_{S}^{2 k}\right) & =\operatorname{vol}\left(\mathbb{S}^{2 k}\right)
\end{aligned}
$$

Above formulas say that the total volume of even dimensional model has unique value for any contour but odd dimensional model has infinitely many values for various types of contours.

Note that we should know that two kinds of contours (clockwise and counterclockwise contour) could be supported and be comprehended by the $\epsilon$ approximation technique using $d_{\epsilon}=1 \pm \epsilon i$. But it is unclear that we can use an appropriate $\epsilon$-approximation technique for other types of contours. So we should make a proper mathematical theory to other contours.

Naturally the Lorentzian isometry group $O(n, 1)$ can be considered as the isometry group of the hyperbolic sphere and spherical sphere. More precisely, we know the following proposition (see [2] for a proof and see [3] for more delicate boundary condition).

Proposition 2.6. Let $U$ be a domain with piecewise analytic boundary transversal to $\partial \mathbb{H}^{n}$ in the extended hyperbolic space. Then $\operatorname{vol}_{H}(U)$ has a well-defined finite value and $\operatorname{vol}_{H}(g(U))=\operatorname{vol}_{H}(U)$ for each $g \in P O(n, 1)$.

In fact, we already know that for a given $g$ in $\operatorname{Isom}\left(\mathbb{H}^{n}\right)$, which is index two subgroup of $O(n, 1)$, and for a given domain $U$ contained in $\mathbb{H}^{n}$, we get the equality $\operatorname{vol}(g(U))=\operatorname{vol}(U)$. Surprisingly the volume of nice domains intersecting with $\partial \mathbb{H}^{n}\left(=\pi\left(L^{n}\right)\right)$ can be calculated. Though each part of the set divided by $\partial \mathbb{H}^{n}$ has infinite volume, the total volume of two parts become finite. This model has three infinite volume parts, $\pi\left(H_{+}^{n}\right), \pi\left(H_{-}^{n}\right)$, and $\pi\left(S_{1}^{n}\right)$, but by summing these parts we can get a finite total volume and hence a finite geometry by using a finitely additive measure theory (see [2]) without any contradiction. 


\section{Length and angle on the extended hyperbolic space and extended de Sitter space}

It is obvious from the definition of its metric that the geodesics on $\mathbb{S}_{H}^{n}$ (resp. $\mathbb{S}_{S}^{n}$ ) are great circles on $\mathbb{S}_{H}^{n}$ (resp. $\mathbb{S}_{S}^{n}$ ) and more generally the totally geodesic subspaces are the intersections of the linear subspaces of $\mathbb{R}^{n, 1}$ with $\mathbb{S}_{H}^{n}$ (resp. $\left.\mathbb{S}_{S}^{n}\right)$ just like on $\mathbb{S}^{n}$ (refer to $[10]$ ).

We denote the distance between two points $A$ and $B$ in the extended hyperbolic space $\mathbb{S}_{H}^{n}$ as $d_{H}(A, B)$. Let's first discuss the distance between two points on $\mathbb{S}_{H}^{1}$. In this case, the formula (3) helps the calculation of the distance of two points in $\mathbb{S}_{H}^{1}$. For instance, if $A$ and $B$ are symmetric with respect to the light cone $x_{0}=x_{1}$ in $\mathbb{R}^{1,1}$ as in Fig. 4 (i.e., $A$ and $B$ as vectors of $\mathbb{R}^{1,1}$ are perpendicular), then their affine coordinates are $a(<1)$ and $\frac{1}{a}$, and the distance will be $\frac{\pi}{2} i$ by formula (3). The distance between isometric images $A^{\prime}$ and $B^{\prime}$ of $A$ and $B$ will be again $\frac{\pi}{2} i$ being symmetric, and hence $d_{H}\left(B, B^{\prime}\right)=-d_{H}\left(A, A^{\prime}\right)$ in Fig. 4.

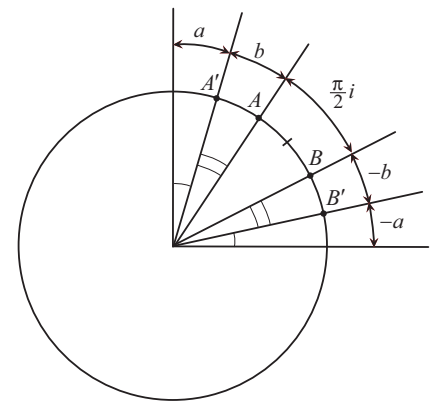

Fig. 4

To discuss the distance between two points in $\mathbb{S}_{H}^{n}$ in general, it suffices to consider on $\mathbb{S}_{H}^{2}$.

For actual computations, it would be convenient to divide into the following 3 cases. For the case when the geodesic connecting two points meet $\partial \mathbb{H}^{2}$ transversely, we may assume that these two points lie on $\mathbb{S}_{H}^{1}=\mathbb{S}_{H}^{2} \cap\left\{x \mid x_{2}=0\right\}$ by an isometry and can handle as discussed above.

For the case when the geodesic line connecting these two points does not intersect $\partial \mathbb{H}^{2}$, we can send this line to the equator $\left(=\mathbb{S}_{H}^{2} \cap\left\{x \mid x_{0}=0\right\}\right)$ of $\mathbb{S}_{H}^{2}$ by an isometry, and hence the distance becomes $i$ times the distance on the standard Euclidean unit circle.

The remaining case is when the line is tangent to $\partial \mathbb{H}^{2}$. We can obtain the distance on the tangent line on $K^{2}$ through a theoretical way, but it needs a subtle $\epsilon$-approximation technique (see [2]). In this paper, we consider the tangent case as a definition for convenience. 
Definition 3.1. For a point $x$ lie on $\partial \mathbb{H}^{2}$ and a dual geodesic $x^{\perp}$, the lengths of the line segments in $x^{\perp}$ are defined by

$$
\begin{aligned}
& d_{H}(w, y)=0, \quad \text { if } w, y \text { are in the same side with respect to } x, \\
& d_{H}(y, z)=\pi i, \quad \text { if } y, z \text { are in the opposite sides with respect to } x, \\
& d_{H}(x, y)=d_{H}(x, z)=\frac{\pi}{2} i
\end{aligned}
$$

See Fig. 5.

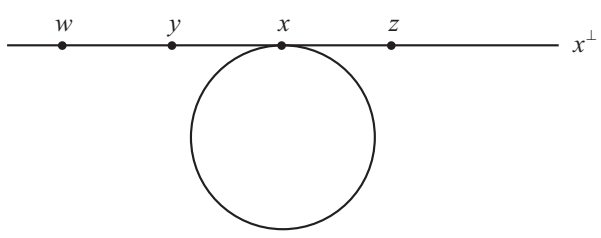

Fig. 5

In all of these discussions, we in fact have to show that 1-dimensional distance is invariant under isometry. That is shown in Theorem 4.3 in [2].

We conclude the following theorem from the above discussion.

Theorem 3.2. The total length of any great circle in $\mathbb{S}_{H}^{n}$ (resp. $\left.\mathbb{S}_{S}^{n}\right)$ is $2 \pi i$ (resp. $2 \pi$ ).

The extended hyperbolic space with Kleinian model has a projective geometric structure, so a geodesic in the model is a straight line and a dual of a point $x$, i.e., $x^{\perp}$ is easily obtained as usual (see Fig. 6 ). Then the length of a geodesic line segment joining $x$ (respectively $y$ ) and an arbitrary point in $x^{\perp}$ (respectively $y^{\perp}$ ) is $\frac{\pi}{2} i$. (Note if the model is considered as a extended de Sitter space, then we should change $\frac{\pi}{2} i$ to $\frac{\pi}{2}$.) This follows since there is an isometry which takes $x$ and $x^{\perp}$ to a point on the equator and to a longitude respectively, and takes $y$ and $y^{\perp}$ to a north pole and to the equator respectively.

Now we define angles on this extended model $\mathbb{S}_{H}^{n}$. From two tangent vectors $v_{p}, w_{p}$ at a point $p$ on a Riemannian part, we can define an angle $\theta$ by the equation

$$
\left\langle v_{p}, w_{p}\right\rangle=\left\|v_{p}\right\|\left\|w_{p}\right\| \cos \theta, \quad 0 \leq \theta \leq \pi .
$$

But for the Lorentzian part, we have some difficulties with this formula since the function $\cos ^{-1}$ is multi-valued and $\theta$ can take several complex values. The definitions of angle have been given through the combinatorial way in [5] and through the cross ratio in [12]. The following definition shows an easy way of defining angle on $\mathbb{S}_{H}^{n}$ and $\mathbb{S}_{S}^{n}$. Note that $v_{p}$ denotes the tangent vector at a point $p \in H_{ \pm}^{n}$ or $S_{1}^{n} \subset \mathbb{R}^{n, 1}$ and $v \in \mathbb{R}^{n, 1}$ independently denotes the parallel translation of $v_{p}$ to origin. 

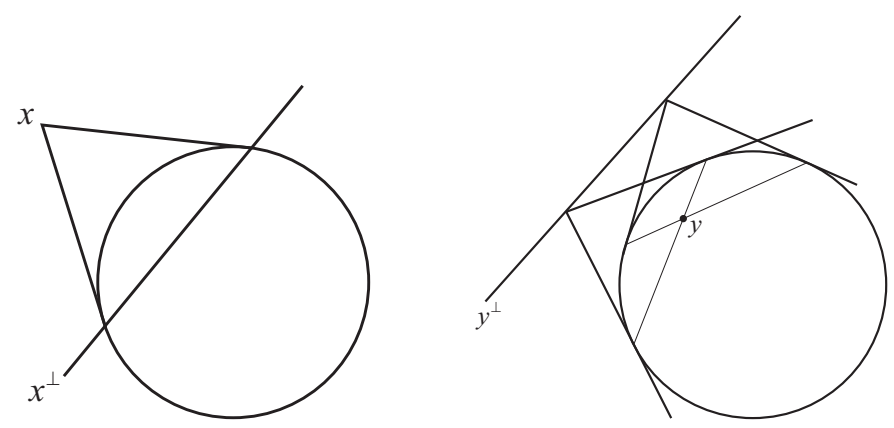

Fig. 6

Definition 3.3. For given two vectors $v, w \in \mathbb{R}^{n, 1}$, the angle between $v$ and $w, \theta=\angle(v, w)$, is defined as $-i \cdot d_{H}(v, w)\left(=d_{S}(v, w)\right)$, where $d_{H}(v, w)$ (resp. $\left.d_{S}(v, w)\right)$ is the length of a geodesic segment joining two points of $\mathbb{S}_{H}^{n}$ (resp. $\left.\mathbb{S}_{S}^{n}\right)$ radially projected from $v, w$ to $\mathbb{S}_{H}^{n}\left(\operatorname{resp} . \mathbb{S}_{S}^{n}\right)$.

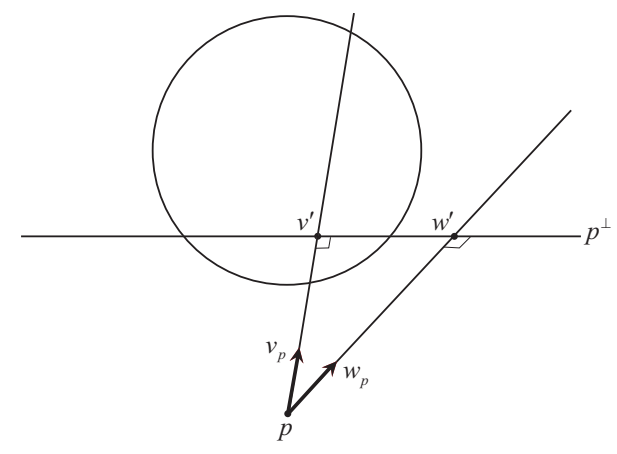

Fig . 7

For $p \in \mathbb{S}_{H}^{n}$ and $p \notin \partial \mathbb{H}^{n}$, the angle $\angle\left(v_{p}, w_{p}\right)$ between two tangent vectors $v_{p}, w_{p} \in T_{p} \mathbb{S}_{H}^{n}$ is defined as $-i \cdot d_{H}\left(v^{\prime}, w^{\prime}\right)$, where $v^{\prime}$ is a point which is obtained by the intersection of the dual plane $p^{\perp}$ and the geodesic line starting at $p$ with direction $v_{p}$ (see Fig. 7).

If $p \in \mathbb{S}_{H}^{2}$ lies on $\partial \mathbb{H}^{2}$, we define the angle $\angle\left(v_{i}, v_{i+1}\right)$ for the tangent vectors $v_{1}, \ldots, v_{5}$ configured as in Fig. 8 as follows:

$$
\angle\left(v_{1}, v_{2}\right)=\angle\left(v_{5}, v_{1}\right)=\frac{\pi}{2}, \angle\left(v_{2}, v_{3}\right)=\angle\left(v_{4}, v_{5}\right)=0 \text {, and } \angle\left(v_{3}, v_{4}\right)=\pi \text {. }
$$

If $p \in \partial \mathbb{H}^{n}$ with $n \geq 3$, we have another type of tangent plane at $p$ which touches $\partial \mathbb{H}^{n}$ at the only point $p$. In this plane, we define the angle at $p$ as the usual Euclidean angle. 


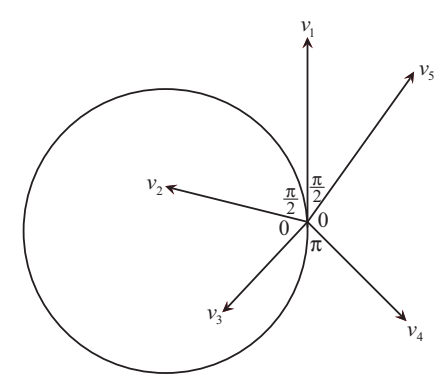

Fig. 8

It is clear that an angle of one rotation around a point is $2 \pi$ by Definition 3.3 and Theorem 3.2. Notice that the factor $-i$ is multiplied to normalize the total length $2 \pi i$ of the great circle in $\mathbb{S}_{H}^{n}$ as $2 \pi$ (see Theorem 3.2).

The isometry invariance of an angle at a point $p \in \mathbb{S}_{H}^{n} \backslash \partial \mathbb{H}^{n}$ is obtained from the invariance of distance.

Remark 3.4. In fact, the second part of Definition 3.3 is obtained from the first part of that, but we made it as a definition for convenience. Even though a justification of the third and fourth part of the definition comes from the $\epsilon$-approximation technique, we only refer the reader to [2].

Definition 3.5. For a lune $l\left(x_{p}, y_{p}\right), \angle\left(x_{p}, y_{p}\right)$ denotes the angle of $l\left(x_{p}, y_{p}\right)$ at the vertex $p$. Here the lune $l\left(x_{p}, y_{p}\right)$ is the inner region generated by two half great circles starting at $p$ with direction $x_{p}, y_{p}$ respectively and ending at $-p$.

A lens $L\left(x^{\perp}, y^{\perp}\right)$ is the intersection of two hemispheres $H_{x}$ and $H_{y}$, where the hemisphere $H_{x}$ is posed opposite to $x$ and $\partial H_{x}$ is perpendicular to $x$, and $\angle\left(x^{\perp}, y^{\perp}\right)$ denotes the dihedral angle of the lens $L\left(x^{\perp}, y^{\perp}\right)$. A lens $L\left(x^{\perp}, y^{\perp}\right)$ is called ideal if $H_{x} \cap H_{y}$ meets the $\partial \mathbb{H}^{n}$ at two points only.

Definition 3.6. For a given lens $L\left(x^{\perp}, y^{\perp}\right)$, the dihedral angle of the lens is defined as the angle $\angle\left(u_{p}, v_{p}\right)$. Here $u_{p}$ (resp. $\left.v_{p}\right)$ is a tangent vector on $\partial H_{x}$ (resp. $\partial H_{y}$ ) with a base point $p \in H_{x} \cap H_{y}$, and $u_{p}, v_{p}$ are perpendicular to $H_{x} \cap H_{y}$. Note that for a non-ideal lens case the vertex $p$ can take any point in $H_{x} \cap H_{y}$, for an ideal lens case the vertex $p$ only can take one of two ideal points in $H_{x} \cap H_{y}$.

Remark 3.7. It is easy to show the well-definedness of the dihedral angle of a lens. In particular for a non-ideal lens, tangent vectors $u_{p}, v_{p}$ are uniquely determined up to positive constant magnitude. But for an ideal lens, tangent vectors $u_{p}, v_{p}$ at an ideal point $p$ can have infinitely many directions.

Since the dihedral angle of a polyhedron can be defined as the dihedral angle of the induced lens naturally. The notion of dihedral angle becomes an important object in the polyhedron theory at the extended hyperbolic space. 
A lune is a 2-dimensional object and a lens in $\mathbb{S}_{H}^{n}$ or $\mathbb{S}_{S}^{n}$ is an $n$-dimensional object.

If a lune $l\left(x_{p}, y_{p}\right)$ with an angle $\theta$ and a lens $L\left(x^{\perp}, y^{\perp}\right)$ with a dihedral angle $\alpha$ are given in $\mathbb{S}_{H}^{2}$ or $\mathbb{S}_{S}^{2}$, then by case by case examinations we get one of the following three kinds of relations (see Fig. 9):

$$
\alpha=\pi-\theta \text { or }-\pi+\theta \text { or } \pi+\theta \text {. }
$$
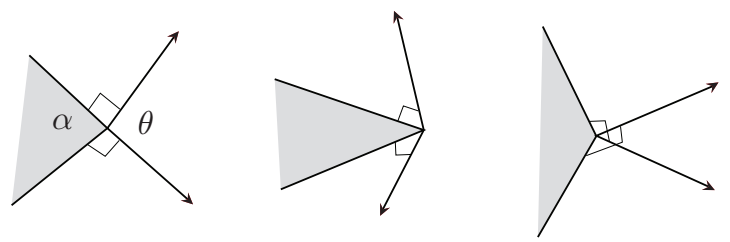

Fig . 9

We already know that the Riemannian case has unique relation $\alpha=\pi-\theta$. For an $n$-dimensional lens, we also conclude the same result as the 2 dimensional case.

Lemma 3.8. For a lens $L\left(x^{\perp}, y^{\perp}\right)$, there are equalities,

$$
\angle\left(x^{\perp}, y^{\perp}\right)= \pm\left(\pi-\angle\left(x_{p}, y_{p}\right)\right) \text { or } \pi+\angle\left(x_{p}, y_{p}\right)
$$

Proof. The dihedral angle of non-ideal lens $L\left(x^{\perp}, y^{\perp}\right)$ is the same as an angle of a lune which is the intersection of the lens $L\left(x^{\perp}, y^{\perp}\right)$ and a 2-dimensional embedded geodesic plane(in fact, a 2 -sphere) generated by a point $p$ and two tangent vectors $x_{p}, y_{p}$, where the point $p$ is an arbitrary point in $x^{\perp} \cap y^{\perp}$. So we can apply the 2-dimensional result to the $n$-dimensional case.

For an ideal lens $L\left(x^{\perp}, y^{\perp}\right)$, we can check the relation $\angle\left(x^{\perp}, y^{\perp}\right)=\pi-$ $\angle\left(x_{p}, y_{p}\right)$.

Remark 3.9. For a given lens with dihedral angle $\alpha=\angle\left(x^{\perp}, y^{\perp}\right)$, if we define an oriented angle $\tilde{\theta}$ and can pass over the $\pi$, then we can unify the three relations to a single relation $\alpha=\pi-\tilde{\theta}$.

The following lemma is given in Thurston's book [13] or [11]. At first we need some notations: The Lorentzian norm of a vector $x$ in $\mathbb{R}^{n, 1}$ is defined to be a complex number

$$
\|x\|=\langle x, x\rangle^{1 / 2},
$$

where $\|x\|$ is either positive, zero, or positive pure imaginary. If $\|x\|$ is positive imaginary, we denote its absolute value by $|\|x\||$. In fact, $\|x\|$ can have minus or minus pure imaginary values, but those choices are not suitable to our clockwise contour convention 2.1.

We have to be cautious about the difference between $\|x\|$ and $\left\|x_{p}\right\|$ for $x \in$ $\mathbb{R}^{n, 1}$. The vector $x$ is parallel translation of the tangent vector $x_{p} \in T_{p} S_{1}^{n}$ or 
$T_{p} H_{+}^{n}$ to the origin. If a point $p$ is contained in the Lorentzian part of $\mathbb{S}_{H}^{n}$ (resp. $\mathbb{S}_{S}^{n}$ ), then we know $\left\|x_{p}\right\|=i\|x\|$ (resp. $\left\|x_{p}\right\|=\|x\|$ ) by Conventions 2.2 and 2.3. Since we changed the sign of the induced metric on the Lorentzian part. Also if a point $p$ is contained in the hyperbolic part of $\mathbb{S}_{H}^{n}\left(\operatorname{resp} . \mathbb{S}_{S}^{n}\right)$, then we know $\left\|x_{p}\right\|=\|x\|$ (resp. $\left\|x_{p}\right\|=-i\|x\|$ ) by Conventions 2.2 and 2.3. Note the sign change of metric induces $\left\langle x_{p}, y_{p}\right\rangle=-\langle x, y\rangle$. Hence we have the identity:

$$
\frac{\left\langle x_{p}, y_{p}\right\rangle}{\left\|x_{p}\right\|\left\|y_{p}\right\|}=\frac{\langle x, y\rangle}{\|x\|\|y\|}
$$

Lemma 3.10 (interpretation of the inner product). If $x$ and $y$ are vectors in $\mathbb{R}^{n, 1}$, then either

(a) $x, y$ are timelike vectors and $\langle x, y\rangle= \pm\|x\|\|y\| \cosh d_{H}( \pm x, y)$; or

(b) $x$ is a timelike vector and $y$ is a spacelike vector, and

$\langle x, y\rangle= \pm \mid\|x\|\|\| y \| \sinh d_{H}\left(x, y^{\perp}\right) ;$ or

(c) $x, y$ are spacelike vectors and the hyperplanes $x^{\perp}, y^{\perp}$ are secant, parallel or ultra parallel depending on whether the intersection $x^{\perp} \cap y^{\perp}$ pass through respectively the inside of $\mathbb{H}^{n}, \partial \mathbb{H}^{n}$ or the outside of $\mathbb{H}^{n}$ only. In the first case, $\langle x, y\rangle=-\|x\|\|y\| \cos \angle\left(x^{\perp}, y^{\perp}\right)$; in the second, $\langle x, y\rangle= \pm\|x\|\|y\|$; and in the third, $\langle x, y\rangle= \pm\|x\|\|y\| \cosh d_{H}\left(x^{\perp}, y^{\perp}\right)$.

The above lemma has many cases for explaining the inner product. However our new notion $d_{H}(x, y)$ in the extended hyperbolic space enables us unify all these cases into a single form as in the following theorem. This shows one good aspect of natural property of the extended space.

Theorem 3.11. For vectors $x$ and $y$ in the Lorentzian space $\mathbb{R}^{n, 1}$ and with condition $d_{H}(x, y) \neq \infty$, we have

$$
\langle x, y\rangle=\|x\|\|y\| \cosh d_{H}(x, y) .
$$

Note that the case $d_{H}(x, y)=\infty$ induces that $\cosh d_{H}(x, y)=\infty,\|x\|$ or $\|y\|=0$ (one of two vectors $x$ and $y$ becomes a lightlike vector), and $\langle x, y\rangle$ take a certain value, hence we get $0 \cdot \infty=$ constant. In some sense, the formula is always true for all cases.

Proof. It is sufficient to consider the following 3 cases by isometric changes. The cases are respectively when the intersection of the plane $\operatorname{span}\{v, w\}$ and $\mathbb{S}_{H}^{n}$ is $\mathbb{S}_{H}^{1}$, the equator $\mathbb{S}_{H}^{2} \cap\left\{x \mid x_{0}=0\right\}$, or the great circle tangent to $\partial \mathbb{H}^{2}$.

By linear property of $\langle\cdot, \cdot\rangle$ and $\|\cdot\|$, and the definition of $d_{H}$, we can assume that $\|x\|,\|y\|=1$ or $i$ or 0 .

For the first case, let's suppose $x=(1,0)$, then if $y$ is timelike vector, then $y$ is represented by $( \pm \cosh a, \sinh a)$; if $y$ is spacelike vector, then $y$ is represented by $( \pm \sinh a, \cosh a)$. Also we should consider two spacelike vectors case $x=(0,1)$ and $y=(\sinh a, \pm \cosh a)$.

Second case induces $x=(0,1,0)$ and $y=(0, \cos a, \sin a)$. 
Third case induces $x=(1,1,0), y=(a, a, 1)$ or $x=(1,1,0), y=(-1,-1,0)$ or $x=(a, a, 1), y=(b, b, 1)$ or $x=(a, a, 1), y=(b, b,-1)$. Note all above $a, b$ are positive numbers.

All cases are checked below.

- $x=(1,0), y=(\cosh a, \sinh a)$ implies $d_{H}(x, y)=d_{H}(0, \tanh a)=$ $\log \sqrt{\frac{1+\tanh a}{1-\tanh a}}$ hence $\|x\|\|y\| \cosh d_{H}(x, y)=i \cdot i \cdot \cosh a=-\cosh a=$ $\langle x, y\rangle$.

- $x=(1,0), y=(-\cosh a, \sinh a)$ implies $d_{H}(x, y)=\pi i-d_{H}(0, \tanh a)$ hence $\|x\|\|y\| \cosh d_{H}(x, y)=i \cdot i \cdot(-\cosh a)=\cosh a=\langle x, y\rangle$.

- $x=(1,0), y=( \pm \sinh a, \cosh a)$ implies $d_{H}(x, y)=\frac{\pi}{2} i \pm d_{H}(0, \tanh a)$ hence $\|x\|\|y\| \cosh d_{H}(x, y)=i \cdot 1 \cdot( \pm i \sinh a)=\mp \sinh a=\langle x, y\rangle$.

- $x=(0,1), y=(\sinh a, \cosh a)$ implies $d_{H}(x, y)=-d_{H}(0, \tanh a)$ hence $\|x\|\|y\| \cosh d_{H}(x, y)=1 \cdot 1 \cdot \cosh a=\cosh a=\langle x, y\rangle$.

- $x=(0,1), y=(\sinh a,-\cosh a)$ implies $d_{H}(x, y)=\pi i+d_{H}(0, \tanh a)$ hence $\|x\|\|y\| \cosh d_{H}(x, y)=1 \cdot 1 \cdot(-\cosh a)=-\cosh a=\langle x, y\rangle$.

- $x=(0,1,0), y=(0, \cos a, \sin a)$ implies $d_{H}(x, y)=a i$ hence $\|x\|\|y\|$ $\cosh d_{H}(x, y)=1 \cdot 1 \cdot \cos a=\cos a=\langle x, y\rangle$.

- $x=(1,1,0), y=(a, a, 1)$ implies $d_{H}(x, y)=\frac{\pi}{2} i$ hence $\|x\|\|y\|$ cosh $d_{H}(x, y)=0 \cdot 1 \cdot \cosh \frac{\pi}{2} i=0=\langle x, y\rangle$.

- $x=(1,1,0), y=(-1,-1,0)$ implies $d_{H}(x, y)=\pi i$ hence $\|x\|\|y\| \cosh$ $d_{H}(x, y)=0 \cdot 0 \cdot \cosh \pi i=0=\langle x, y\rangle$.

- $x=(a, a, 1), y=(b, b, 1)$ implies $d_{H}(x, y)=0$ hence $\|x\|\|y\|$ cosh $d_{H}(x, y)=1 \cdot 1 \cdot \cosh 0=1=\langle x, y\rangle$.

- $x=(a, a, 1), y=(b, b,-1)$ implies $d_{H}(x, y)=\pi i$ hence $\|x\|\|y\|$ cosh $d_{H}(x, y)=1 \cdot 1 \cdot \cosh \pi i=-1=\langle x, y\rangle$.

Now we have examined all the cases and complete the proof.

Here we do not use the result of Lemma 3.10 in the proof of Theorem 3.11. However we can prove Theorem 3.11 from Lemma 3.10. Conversely, we can prove Lemma 3.10 from Theorem 3.11.

Corollary 3.12. For vectors $x$ and $y$ in the Lorentzian space $\mathbb{R}^{n, 1}$ and a point $p$ in $x^{\perp} \cap y^{\perp}$ and with condition $d_{H}(x, y) \neq \infty$, we have

$$
\begin{gathered}
\langle x, y\rangle=\|x\|\|y\| \cos \angle(x, y), \\
\left\langle x_{p}, y_{p}\right\rangle=\left\|x_{p}\right\|\left\|y_{p}\right\| \cos \angle\left(x_{p}, y_{p}\right), \\
\langle x, y\rangle=-\|x\|\|y\| \cos \angle\left(x^{\perp}, y^{\perp}\right), \\
\langle x, y\rangle=\|x\|\|y\| \cos d_{S}(x, y) .
\end{gathered}
$$

Proof. See Lemma 3.8 and Definition 3.3.

Corollary 3.12 shows that the hyperbolic sphere $\mathbb{S}_{H}^{n}$ and the spherical sphere $\mathbb{S}_{S}^{n}$ and the definitions about length and angle on the spaces have natural and essential properties. 
We already showed $\langle x, y\rangle=\|x\|\|y\| \cos \angle(x, y)$ from Definition 3.3. If we add the following three properties to the formula (5), then we can show that the angle is uniquely determined by these four properties and equivalent to Definition 3.3. The additional three properties are

(i) the invariance under isometry,

(ii) finite additivity of angle: if $\theta$ consists of two parts $\theta_{1}$ and $\theta_{2}$, then $\theta=\theta_{1}+\theta_{2}$,

(iii) the angle of half rotation is $\pi$, i.e., a straight line has angle $\pi$.

Other equivalent angle definitions are shown at Remark 4.13 in [2].

Finally we remark that $\theta=\frac{\pi}{2}$ implies $\langle x, y\rangle=0$, but $\langle x, y\rangle=0$ does not imply $\theta=\frac{\pi}{2}$.

\section{Cosine laws and sine law for general triangles}

\subsection{Cosine laws}

In the hyperbolic space, cosine laws and sine law are basic laws as well as the spherical space. So we have to examine whether these laws are satisfied in the extended hyperbolic space.

We need some definitions.

Definition 4.1. For a negative real number $a, \sqrt{a}$ means $\sqrt{-a} i$.

For example, $\sqrt{4}=2$ and $\sqrt{-4}=2 i$.

Definition 4.2. For a complex number $a \in(\mathbb{R} \cup \mathbb{R} i)-\{0\}, \operatorname{sgn}(a)$ is defined as follows:

$$
\operatorname{sgn}(a)=\left\{\begin{aligned}
1 & \text { if } a \text { is positive or positive pure imaginary } \\
-1 & \text { if } a \text { is negative or negative pure imaginary }
\end{aligned}\right.
$$

The sgn notation is slightly generalized, so the usual properties are not satisfied any more. For example, $\operatorname{sgn}(a b)=\operatorname{sgn}(a) \operatorname{sgn}(b)$ is not satisfied, if both of $a$ and $b$ are pure imaginary numbers.

Definition 4.3. For non-zero real numbers $a_{1}, a_{2}, \ldots, a_{n}$, the function msgn (many elements sign) is defined by

$$
\operatorname{msgn}\left(a_{1}, a_{2}, \ldots, a_{n}\right)=\frac{\sqrt{a_{1}} \sqrt{a_{2}} \cdots \sqrt{a_{n}}}{\sqrt{a_{1} a_{2} \cdots a_{n}}} .
$$

From the Definition 4.3, we easily obtain the following proposition.

Proposition 4.4. For non-zero real numbers $a, a_{1}, \ldots, a_{n}, b_{1}, \ldots, b_{m}$, we obtain

(a) $\operatorname{msgn}(a)=1$,

(b) $\operatorname{msgn}(a, a)=\operatorname{sgn}(a)$, and $\operatorname{msgn}\left(a_{1}, a_{2}, \ldots, a_{n}\right) \operatorname{msgn}\left(a_{1}, a_{2}, \ldots, a_{n}\right)=1$,

(c) $\operatorname{msgn}\left(a_{1}, a_{2}, \ldots, a_{n}\right) \operatorname{msgn}\left(b_{1}, b_{2}, \ldots, b_{m}\right)$

$$
=\operatorname{msgn}\left(a_{1}, \ldots, a_{n}, b_{1}, \ldots, b_{m}\right) \operatorname{msgn}\left(a_{1} \cdots a_{n}, b_{1} \cdots b_{m}\right) \text {, }
$$


(d) $\operatorname{msgn}\left(a_{1}, a_{2}, \ldots, a_{n}\right)=(-1)^{\left[\frac{\alpha}{2}\right]}$, where [.] is the Gauss notation and $\alpha$ is the number of negative elements among $a_{i}, i=1, \ldots, n$,

(e) $\operatorname{msgn}\left(a_{1}, a_{1}, a_{2}, a_{2}, \ldots, a_{n}, a_{n}\right)=\operatorname{sgn}\left(a_{1} a_{2} \cdots a_{n}\right)$.

Proof. All of these follows easily from Definition 4.3.

We also need next definitions to prove the cosine and sine laws.

Definition 4.5. (1) For a given hemisphere $H \in \mathbb{S}_{H}^{n}$ (resp. vector $v \in \mathbb{S}_{H}^{n}$ ), the algebraic dual of $H$ (resp. $v$ ) is a point $v$ (resp. hemisphere $H$ ) given by $\langle H, v\rangle \geq 0$, i.e., ${ }^{\forall} h \in H\langle h, v\rangle \geq 0$.

(2) For a given hemisphere $H \in \mathbb{S}_{H}^{n}$ (resp. vector $v \in \mathbb{S}_{H}^{n}$ ), the geometric dual of $H$ (resp. $v$ ) is a point $v$ (resp. hemisphere $H$ ) given by $\langle\partial H, v\rangle=0$ and $v \notin H$.

For convenience sake, we denote an algebraic (resp. geometric) dual of $X$ as $X^{a \perp}$ (resp. $X^{g \perp}$ ).

Remark 4.6. For a given ideal (i.e., tangent to $\partial \mathbb{H}^{n}$ ) hemisphere $H$, the algebraic dual vector $v=H^{a \perp}$ is well defined. But the geometric dual vector $v=H^{g \perp}$ is not well defined and there are two direction choices (i.e., two points in $\mathbb{S}_{H}^{n}$ ) for the vector $v$.

Definition 4.7. For given three linearly independent non-lightlike vectors $v_{1}$, $v_{2}, v_{3}$ (resp. non-ideal hemispheres $H_{1}, H_{2}, H_{3}$ ) in $\mathbb{S}_{H}^{2}$, the three vectors (resp. hemispheres) induce a unique triangle $\triangle\left(v_{1}, v_{2}, v_{3}\right)$ (resp. $\triangle\left(H_{1}, H_{2}, H_{3}\right)$ ) with sides composed of "smaller" geodesics (resp. $H_{1} \cap H_{2} \cap H_{3}$ ). Then the algebraic (resp. geometric) dual of triangle $\triangle=\triangle\left(H_{1}, H_{2}, H_{3}\right)$ is a triangle obtained by three points $H_{1}^{a \perp}, H_{2}^{a \perp}, H_{3}^{a \perp}$ (resp. $H_{1}^{g \perp}, H_{2}^{g \perp}, H_{3}^{g \perp}$ ), and is denoted as $\triangle\left(H_{1}, H_{2}, H_{3}\right)^{a \perp}$ or $\triangle^{a \perp}$ (resp. $\triangle\left(H_{1}, H_{2}, H_{3}\right)^{g \perp}$ or $\left.\triangle^{g \perp}\right)$.

Remark 4.8. There are two geodesic segments joining $v$ and $w(\neq-v)$ in $\mathbb{S}_{H}^{n}$. We can choose one geodesic segment "smaller" than the other. Here the meaning of "smaller" is not smaller in length (because the length in this model has complex value) but the one which does not contain two antipodal points.

The following corollary is an easy consequence of the above definitions.

Corollary 4.9. For a triangle in $\mathbb{S}_{H}^{2}$,

(1) $\triangle\left(v_{1}, v_{2}, v_{3}\right)^{a \perp}=\triangle\left(v_{1}^{a \perp}, v_{2}^{a \perp}, v_{3}^{a \perp}\right)$.

(2) $\left(\triangle^{a \perp}\right)^{a \perp}=\triangle$.

Remark 4.10. The relation $\left(\triangle^{g \perp}\right)^{g \perp}=\triangle$ is not satisfied in general. In order to get the relation $\left(\triangle^{g \perp}\right)^{g \perp}=\triangle$, we have to find a different type triangle edge and interior construction for the definition of $\triangle^{g \perp}$ with the same three vertices.

Now we can calculate the trigonometric formulas for a triangle in $\mathbb{S}_{H}^{2}$. We start with any linearly independent non-lightlike triple $\left(v_{1}, v_{2}, v_{3}\right)$ of vectors in $\mathbb{R}^{2,1}$. They determine a triangle $\triangle\left(v_{1}, v_{2}, v_{3}\right)$ formed by smaller geodesics. The 
dual basis of $\left(v_{1}, v_{2}, v_{3}\right)$ is another triple $\left(w_{1}, w_{2}, w_{3}\right)$ of vectors in $\mathbb{R}^{2,1}$, defined by the conditions $\left\langle v_{i}, w_{i}\right\rangle=1$ and $\left\langle v_{i}, w_{j}\right\rangle=0$ if $i \neq j$ for $i, j=1,2,3$. Thus we have $\triangle\left(v_{1}, v_{2}, v_{3}\right)^{a \perp}=\triangle\left(w_{1}, w_{2}, w_{3}\right)$. If we let $V$ and $W$ be the matrices with columns $v_{i}$ and $w_{i}$, then they satisfy the equation $W^{t} S V=I$, where $S$ is a diagonal matrix with entries $(-1,1,1)$. However the matrices of inner product, $V^{t} S V$ and $W^{t} S W$, are still inverse to each other:

$$
\left(V^{t} S V\right)\left(W^{t} S W\right)=\left(V^{t} S V\right)\left(V^{-1} W\right)=V^{t} S W=\left(W^{t} S V\right)^{t}=I .
$$

The matrix $V^{t} S V$ can be written as

$$
V^{t} S V=\left(\begin{array}{lll}
c_{11} & c_{12} & c_{13} \\
c_{12} & c_{22} & c_{23} \\
c_{13} & c_{23} & c_{33}
\end{array}\right), \quad c_{i j}=\left\langle v_{i}, v_{j}\right\rangle
$$

and hence $W^{t} S W$ is represented as

(6)

$$
W^{t} S W=\frac{1}{\operatorname{det}\left(V^{t} S V\right)}\left(\begin{array}{ccc}
c_{22} c_{33}-c_{23}^{2} & c_{13} c_{23}-c_{33} c_{12} & c_{12} c_{23}-c_{22} c_{13} \\
c_{13} c_{23}-c_{33} c_{12} & c_{11} c_{33}-c_{13}^{2} & c_{12} c_{13}-c_{11} c_{23} \\
c_{12} c_{23}-c_{22} c_{13} & c_{12} c_{13}-c_{11} c_{23} & c_{11} c_{22}-c_{12}^{2}
\end{array}\right) .
$$

We need another notation. Let's denote the geometric dual of $\triangle\left(v_{1}, v_{2}, v_{3}\right)$ as $\triangle\left(w_{1}^{\prime}, w_{2}^{\prime}, w_{3}^{\prime}\right)$. Then the formula (5) in Corollary 3.12 gives the angle $\theta$ of vertex $v_{3}$ as

$$
\left\langle w_{1}^{\prime}, w_{2}^{\prime}\right\rangle=-\left\|w_{1}^{\prime}\right\|\left\|w_{2}^{\prime}\right\| \cos \theta .
$$

Here the $w_{i}$ and $w_{i}^{\prime}$ are the same or differ by -1 , and the difference of $\frac{\left\langle w_{1}^{\prime}, w_{2}^{\prime}\right\rangle}{\left\|w_{1}^{\prime}\right\|\left\|w_{2}^{\prime}\right\|}$ and $\frac{\left\langle w_{1}, w_{2}\right\rangle}{\left\|w_{1}\right\|\left\|w_{2}\right\|}$ is determined by $\operatorname{sgn}\left(\left\|w_{1}\right\|^{2}\left\|w_{2}\right\|^{2}\right)$. Since we get $w_{i}=w_{i}^{\prime}$ if $\left\|w_{i}\right\|^{2}<0$, and $w_{i}=-w_{i}^{\prime}$ if $\left\|w_{i}\right\|^{2}>0$ from Definition 4.5 or simply

$$
w_{i}=\operatorname{sgn}\left(-\left\|w_{i}\right\|^{2}\right) w_{i}^{\prime} .
$$

From equation (6), we have

$$
\begin{aligned}
& \frac{\left\langle w_{1}, w_{2}\right\rangle}{\left\|w_{1}\right\|\left\|w_{2}\right\|}=\frac{c_{13} c_{23}-c_{33} c_{12}}{\operatorname{det}\left(V^{t} S V\right)} /\left(\sqrt{\frac{c_{22} c_{33}-c_{23}^{2}}{\operatorname{det}\left(V^{t} S V\right)}} \sqrt{\frac{c_{11} c_{33}-c_{13}^{2}}{\operatorname{det}\left(V^{t} S V\right)}}\right) \text {, } \\
& =\operatorname{sgn}\left(\left(c_{22} c_{33}-c_{23}^{2}\right)\left(c_{11} c_{33}-c_{13}^{2}\right)\right) \frac{c_{13} c_{23}-c_{33} c_{12}}{\sqrt{c_{22} c_{33}-c_{23}^{2}} \sqrt{c_{11} c_{33}-c_{13}^{2}}}, \\
& =\operatorname{sgn}\left(\left\|w_{1}\right\|^{2}\left\|w_{2}\right\|^{2}\right) \frac{c_{13} c_{23}-c_{33} c_{12}}{\sqrt{c_{22} c_{33}-c_{23}^{2}} \sqrt{c_{11} c_{33}-c_{13}^{2}}},
\end{aligned}
$$

where we used the fact that $\operatorname{det}\left(V^{t} S V\right)$ is negative and $\sqrt{\bar{\Xi}}=\frac{\sqrt{-}}{\sqrt{-}}, \sqrt{\frac{ \pm}{-}}=$ $-\frac{\sqrt{t}}{\sqrt{-}}$. Therefore we conclude

$$
-\cos \theta=\frac{c_{13} c_{23}-c_{33} c_{12}}{\sqrt{c_{22} c_{33}-c_{23}^{2}} \sqrt{c_{11} c_{33}-c_{13}^{2}}} .
$$


Also we know that $c_{i j}=\left\langle v_{i}, v_{j}\right\rangle=\left\|v_{i}\right\|\left\|v_{j}\right\| \cosh d_{H}\left(v_{i}, v_{j}\right)$ and simply $c_{i j}=$ $\left\|v_{i}\right\|\left\|v_{j}\right\| \cosh d_{i j}$ from Theorem 3.11. So we get

$$
\begin{aligned}
\cos \theta= & \frac{\left\|v_{1}\right\|\left\|v_{2}\right\|\left\|v_{3}\right\|^{2}\left(\cosh d_{12}-\cosh d_{13} \cosh d_{23}\right)}{\sqrt{\left\|v_{1}\right\|^{2}\left\|v_{3}\right\|^{2}\left(1-\cosh ^{2} d_{13}\right)} \sqrt{\left\|v_{2}\right\|^{2}\left\|v_{3}\right\|^{2}\left(1-\cosh ^{2} d_{23}\right)}}, \\
= & \frac{\left\|v_{1}\right\|\left\|v_{2}\right\|\left\|v_{3}\right\|^{2}\left(\cosh d_{12}-\cosh d_{13} \cosh d_{23}\right)}{\sqrt{-\left\|v_{1}\right\|^{2}\left\|v_{3}\right\|^{2} \sinh ^{2} d_{13}} \sqrt{-\left\|v_{2}\right\|^{2}\left\|v_{3}\right\|^{2} \sinh ^{2} d_{23}}}, \\
= & \operatorname{msgn}\left(-1,\left\|v_{1}\right\|^{2},\left\|v_{3}\right\|^{2},\left\|v_{1}\right\|^{2}\left\|w_{2}\right\|^{2}\left\|v_{3}\right\|^{2}\right) \operatorname{msgn}\left(-1,\left\|v_{2}\right\|^{2},\left\|v_{3}\right\|^{2},\right. \\
& \left.\left\|w_{1}\right\|^{2}\left\|v_{2}\right\|^{2}\left\|v_{3}\right\|^{2}\right) \frac{\cosh d_{13} \cosh d_{23}-\cosh d_{12}}{\sqrt{\sinh ^{2} d_{13}} \sqrt{\sinh ^{2} d_{23}}}, \\
= & \operatorname{msgn}\left(-1,\left\|v_{1}\right\|^{2},\left\|v_{3}\right\|^{2},\left\|v_{1}\right\|^{2}\left\|w_{2}\right\|^{2}\left\|v_{3}\right\|^{2}\right) \operatorname{msgn}\left(-1,\left\|v_{2}\right\|^{2},\left\|v_{3}\right\|^{2},\right. \\
& \left.\left\|w_{1}\right\|^{2}\left\|v_{2}\right\|^{2}\left\|v_{3}\right\|^{2}\right) \operatorname{sgn}\left(\sinh d_{12}\right) \operatorname{sgn}\left(\sinh d_{23}\right) \frac{\cosh d_{13} \cosh d_{23}-\cosh d_{12}}{\sinh d_{13} \sinh d_{23}},
\end{aligned}
$$

by considering $\left\|w_{1}\right\|^{2} \operatorname{det}\left(V^{t} S V\right)=-\left\|v_{2}\right\|^{2}\left\|v_{3}\right\|^{2} \sinh ^{2} d_{23}$ and

$$
\left\|w_{2}\right\|^{2} \operatorname{det}\left(V^{t} S V\right)=-\left\|v_{1}\right\|^{2}\left\|v_{3}\right\|^{2} \sinh ^{2} d_{13} .
$$

In the above, the function sgn is defined for pure imaginary number (for example, $\operatorname{sgn}(i)=1$ and $\operatorname{sgn}(-i)=-1)$, and $\operatorname{sgn}\left(\sinh d_{23}\right)$ is negative if and only if $\left\|v_{2}\right\|^{2}>0,\left\|v_{3}\right\|^{2}>0$, and $\left\|w_{1}\right\|^{2}>0$. Then we can show the following relations by case by case examination.

$$
\begin{aligned}
\operatorname{sgn}\left(\sinh d_{13}\right) & =-\operatorname{msgn}\left(-1,-\left\|v_{1}\right\|^{2},-\left\|v_{3}\right\|^{2},-\left\|w_{2}\right\|^{2}\right) \\
& =\operatorname{sgn}\left(-\left\|v_{1}\right\|^{3}\left\|v_{3}\right\|^{3}\left\|w_{2}\right\|^{3}\right) \quad \text { and } \\
\operatorname{sgn}\left(\sinh d_{23}\right) & =-\operatorname{msgn}\left(-1,-\left\|v_{2}\right\|^{2},-\left\|v_{3}\right\|^{2},-\left\|w_{1}\right\|^{2}\right) \\
& =\operatorname{sgn}\left(-\left\|v_{2}\right\|^{3}\left\|v_{3}\right\|^{3}\left\|w_{1}\right\|^{3}\right) .
\end{aligned}
$$

The right hand side of the equality (8) has also negative sign -1 , when $\left\|v_{1}\right\|^{2}<0,\left\|v_{3}\right\|^{2}<0$, and $\left\|w_{2}\right\|^{2}<0$. But we need not worry about this, because $\left\|v_{1}\right\|^{2}<0,\left\|v_{3}\right\|^{2}<0$ implies $\left\|w_{2}\right\|^{2}>0$. Hence the case does not exist. So the relations (8) are true statements. Therefore we have to simplify the expression:

$$
\begin{aligned}
& \operatorname{msgn}\left(-1,\left\|v_{1}\right\|^{2},\left\|v_{3}\right\|^{2},\left\|v_{1}\right\|^{2}\left\|w_{2}\right\|^{2}\left\|v_{3}\right\|^{2}\right) \operatorname{msgn}\left(-1,\left\|v_{2}\right\|^{2},\left\|v_{3}\right\|^{2},\left\|w_{1}\right\|^{2}\left\|v_{2}\right\|^{2}\left\|v_{3}\right\|^{2}\right) \\
& \quad \times \operatorname{msgn}\left(-1,-\left\|v_{1}\right\|^{2},-\left\|v_{3}\right\|^{2},-\left\|w_{2}\right\|^{2}\right) \operatorname{msgn}\left(-1,-\left\|v_{2}\right\|^{2},-\left\|v_{3}\right\|^{2},-\left\|w_{1}\right\|^{2}\right) .
\end{aligned}
$$

\section{Lemma 4.11.}

$$
\begin{aligned}
& \operatorname{msgn}\left(-1,\left\|v_{1}\right\|^{2},\left\|v_{3}\right\|^{2},\left\|v_{1}\right\|^{2}\left\|w_{2}\right\|^{2}\left\|v_{3}\right\|^{2}\right) \operatorname{msgn}\left(-1,\left\|v_{2}\right\|^{2},\left\|v_{3}\right\|^{2},\left\|w_{1}\right\|^{2}\left\|v_{2}\right\|^{2}\left\|v_{3}\right\|^{2}\right) \\
& \quad \times \operatorname{msgn}\left(-1,-\left\|v_{1}\right\|^{2},-\left\|v_{3}\right\|^{2},-\left\|w_{2}\right\|^{2}\right) \operatorname{msgn}\left(-1,-\left\|v_{2}\right\|^{2},-\left\|v_{3}\right\|^{2},-\left\|w_{1}\right\|^{2}\right)=1 .
\end{aligned}
$$


Proof. By using Proposition 4.4, we see

$$
\begin{aligned}
& \operatorname{msgn}\left(-1,\left\|v_{2}\right\|^{2},\left\|v_{3}\right\|^{2},\left\|w_{1}\right\|^{2}\left\|v_{2}\right\|^{2}\left\|v_{3}\right\|^{2}\right) \operatorname{msgn}\left(-1,-\left\|v_{2}\right\|^{2},-\left\|v_{3}\right\|^{2},-\left\|w_{1}\right\|^{2}\right) \\
= & \operatorname{msgn}\left(-1,-1,\left\|v_{2}\right\|^{2},-\left\|v_{2}\right\|^{2},\left\|v_{3}\right\|^{2},-\left\|v_{3}\right\|^{2},\left\|w_{1}\right\|^{2}\left\|v_{2}\right\|^{2}\left\|v_{3}\right\|^{2},-\left\|w_{1}\right\|^{2}\right) \\
& \times \operatorname{msgn}\left(-\left\|w_{1}\right\|^{2},\left\|w_{1}\right\|^{2}\left\|v_{2}\right\|^{2}\left\|v_{3}\right\|^{2}\right) \\
= & \left(\operatorname{msgn}\left(-\left\|w_{1}\right\|^{2},\left\|w_{1}\right\|^{2}\left\|v_{2}\right\|^{2}\left\|v_{3}\right\|^{2}\right)\right)^{2} \\
= & 1 .
\end{aligned}
$$

and similarly

$\operatorname{msgn}\left(-1,\left\|v_{1}\right\|^{2},\left\|v_{3}\right\|^{2},\left\|v_{1}\right\|^{2}\left\|w_{2}\right\|^{2}\left\|v_{3}\right\|^{2}\right) \operatorname{msgn}\left(-1,-\left\|v_{1}\right\|^{2},-\left\|v_{3}\right\|^{2},-\left\|w_{2}\right\|^{2}\right)=1$.

As is shown, we conclude

$$
\cos \theta=\frac{\cosh d_{13} \cosh d_{23}-\cosh d_{12}}{\sinh d_{13} \sinh d_{23}} .
$$

Letting $A, B, C$ stand for the angles at $v_{1}, v_{2}, v_{3}$ and $a, b, c$ for the extended hyperbolic lengths of opposite sides, we obtain the hyperbolic law of cosine on the hyperbolic sphere $\mathbb{S}_{H}^{2}$ :

$$
\cos C=\frac{\cosh a \cosh b-\cosh c}{\sinh a \sinh b} .
$$

Also we can easily deduce the spherical law of cosine on the spherical sphere $\mathbb{S}_{S}^{2}$ by using $i \cdot d_{S}=d_{H}$ and so $\cosh d_{H}=\cos d_{S}, \sinh d_{H}=i \sin d_{S}$, where $a, b, c$ represent the extended spherical length,

$$
\begin{aligned}
\cos C & =\frac{\cosh (a i) \cosh (b i)-\cosh (c i)}{\sinh (a i) \sinh (b i)}, \\
& =\frac{\cos c-\cos a \cos b}{\sin a \sin b} .
\end{aligned}
$$

To obtain the dual cosine law, we start our argument from a triangle $\triangle\left(v_{1}\right.$, $\left.v_{2}, v_{3}\right)$ with its geometric dual $\triangle^{g \perp}$ written by $\triangle\left(w_{1}, w_{2}, w_{3}\right)$. In the proof of cosine law, $\triangle\left(w_{1}, w_{2}, w_{3}\right)$ means an algebraic dual, but from now $\triangle\left(w_{1}, w_{2}, w_{3}\right)$ denotes a geometric dual for convenience. The angles and edges of $\triangle$ and $\triangle^{g \perp}$ are shown in Fig. 10.

Lemma 3.8 and Definition 3.3 deduce the relations $-\cos A=\cosh a^{\prime},-\cos B$ $=\cosh b^{\prime}$, and $-\cos C=\cosh c^{\prime}$, but do not gives the relations $-\cos A^{\prime}=$ $\cosh a,-\cos B^{\prime}=\cosh b$, and $-\cos C^{\prime}=\cosh c$ by Remark 4.10. By comparison of $\triangle^{a \perp}$ and $\triangle^{g \perp}$, and comparison of $\left(\triangle^{a \perp}\right)^{g \perp}$ and $\left(\triangle^{a \perp}\right)^{a \perp}=\triangle$, and the relation (7), we can get the exact relations between $\cos A^{\prime}, \cos B^{\prime}, \cos C^{\prime}$ and $\cosh a, \cosh b, \cosh c$ :

$$
\begin{aligned}
& -\cos A^{\prime}=\cosh a \operatorname{sgn}\left(\left\|v_{2}\right\|^{2}\left\|v_{3}\right\|^{2}\left\|w_{2}\right\|^{2}\left\|w_{3}\right\|^{2}\right), \\
& -\cos B^{\prime}=\cosh b \operatorname{sgn}\left(\left\|v_{1}\right\|^{2}\left\|v_{3}\right\|^{2}\left\|w_{1}\right\|^{2}\left\|w_{3}\right\|^{2}\right), \\
& -\cos C^{\prime}=\cosh c \operatorname{sgn}\left(\left\|v_{1}\right\|^{2}\left\|v_{2}\right\|^{2}\left\|w_{1}\right\|^{2}\left\|w_{2}\right\|^{2}\right) .
\end{aligned}
$$




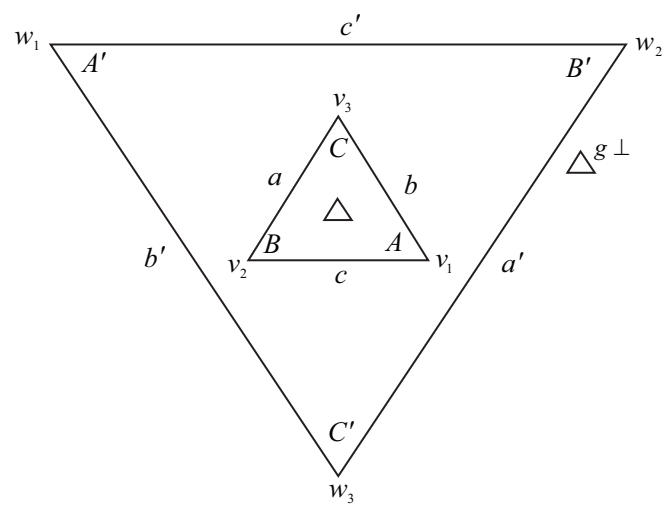

Fig. 10

We already get the cosine law which is adapted to the triangle $\triangle^{g \perp}$ :

$$
\cos C^{\prime}=\frac{\cosh a^{\prime} \cosh b^{\prime}-\cosh c^{\prime}}{\sinh a^{\prime} \sinh b^{\prime}} .
$$

The formula (9) is changed to

$$
-\cosh c \operatorname{sgn}\left(\left\|v_{1}\right\|^{2}\left\|v_{2}\right\|^{2}\left\|w_{1}\right\|^{2}\left\|w_{2}\right\|^{2}\right)=\frac{\cos A \cos B+\cos C}{\sinh a^{\prime} \sinh b^{\prime}},
$$

and we have to show that

$$
\sin A \sin B=-\sinh a^{\prime} \sinh b^{\prime} \operatorname{sgn}\left(\left\|v_{1}\right\|^{2}\left\|v_{2}\right\|^{2}\left\|w_{1}\right\|^{2}\left\|w_{2}\right\|^{2}\right)
$$

in order to obtain the dual cosine law,

$$
\cosh c=\frac{\cos A \cos B+\cos C}{\sin A \sin B} .
$$

The above formula (10) is also changed to

$$
\begin{aligned}
& \sin A \sin B \operatorname{sgn}\left(\left\|v_{1}\right\|^{2}\left\|v_{2}\right\|^{2}\left\|w_{1}\right\|^{2}\left\|w_{2}\right\|^{2}\right) \\
= & -\operatorname{sgn}\left(\sinh a^{\prime}\right) \operatorname{sgn}\left(\sinh b^{\prime}\right) \sqrt{\sinh ^{2} a^{\prime}} \sqrt{\sinh ^{2} b^{\prime}} \\
= & -\operatorname{sgn}\left(\sinh a^{\prime}\right) \operatorname{sgn}\left(\sinh b^{\prime}\right) \sqrt{-1+\cos ^{2} A} \sqrt{-1+\cos ^{2} B} \\
= & \operatorname{sgn}\left(\sinh a^{\prime}\right) \operatorname{sgn}\left(\sinh b^{\prime}\right) \operatorname{msgn}\left(-1, \sin ^{2} A\right) \operatorname{msgn}\left(-1, \sin ^{2} B\right) \sqrt{\sin ^{2} A} \sqrt{\sin ^{2} B},
\end{aligned}
$$

hence we need

$$
\begin{aligned}
& \operatorname{sgn}\left(\left\|v_{1}\right\|^{2}\left\|v_{2}\right\|^{2}\left\|w_{1}\right\|^{2}\left\|w_{2}\right\|^{2}\right) \\
= & \operatorname{sgn}\left(\sinh a^{\prime}\right) \operatorname{sgn}\left(\sinh b^{\prime}\right) \operatorname{msgn}\left(-1, \sin ^{2} A\right) \operatorname{msgn}\left(-1, \sin ^{2} B\right) \operatorname{sgn}(\sin A) \operatorname{sgn}(\sin B) .
\end{aligned}
$$

For complex numbers $z_{1}$ and $z_{2}$, if there exists a positive number $\alpha$ such that $z_{1}=\alpha z_{2}$, then let's denote simply as $z_{1} \sim z_{2}$. Then by easy checking, we 
know $\sinh a^{\prime} \sim-\left\|v_{1}\right\|^{3}\left\|w_{2}\right\|^{3}\left\|w_{3}\right\|^{3}$ and $\sin A \sim-i\left\|v_{1}\right\|\left\|w_{2}\right\|\left\|w_{3}\right\|$. Also we can easily find

$$
\begin{aligned}
\operatorname{sgn}\left(-\left\|v_{1}\right\|^{3}\left\|w_{2}\right\|^{3}\left\|w_{3}\right\|^{3}\right) & =\operatorname{sgn}\left(-i\left\|v_{1}\right\|\left\|w_{2}\right\|\left\|w_{3}\right\|\right) \\
& =-\operatorname{msgn}\left(-1,-\left\|v_{1}\right\|^{2},-\left\|w_{2}\right\|^{2},-\left\|w_{3}\right\|^{2}\right) .
\end{aligned}
$$

Therefore we can get the following identities:

$$
\begin{aligned}
& \operatorname{sgn}\left(\sinh a^{\prime}\right)=\operatorname{sgn}(\sin A)=-\operatorname{msgn}\left(-1,-\left\|v_{1}\right\|^{2},-\left\|w_{2}\right\|^{2},-\left\|w_{3}\right\|^{2}\right), \\
& \operatorname{sgn}\left(\sinh b^{\prime}\right)=\operatorname{sgn}(\sin B)=-\operatorname{msgn}\left(-1,-\left\|v_{2}\right\|^{2},-\left\|w_{1}\right\|^{2},-\left\|w_{3}\right\|^{2}\right),
\end{aligned}
$$

and

$$
\begin{aligned}
& \operatorname{sgn}\left(\sin ^{2} A\right)=\operatorname{sgn}\left(-\left\|v_{1}\right\|^{2}\left\|w_{2}\right\|^{2}\left\|w_{3}\right\|^{2}\right), \\
& \operatorname{sgn}\left(\sin ^{2} B\right)=\operatorname{sgn}\left(-\left\|v_{2}\right\|^{2}\left\|w_{1}\right\|^{2}\left\|w_{3}\right\|^{2}\right) .
\end{aligned}
$$

The only thing left to show is the following lemma.

\section{Lemma 4.12.}

$$
\begin{aligned}
& \operatorname{msgn}\left(-1,-\left\|v_{1}\right\|^{2}\left\|w_{2}\right\|^{2}\left\|w_{3}\right\|^{2}\right) \operatorname{msgn}\left(-1,-\left\|v_{2}\right\|^{2}\left\|w_{1}\right\|^{2}\left\|w_{3}\right\|^{2}\right) \\
= & \operatorname{sgn}\left(\left\|v_{1}\right\|^{2}\left\|v_{2}\right\|^{2}\left\|w_{1}\right\|^{2}\left\|w_{2}\right\|^{2}\right) .
\end{aligned}
$$

Proof. The left hand side of the above equality is equal to

$$
\begin{aligned}
& \operatorname{msgn}\left(-1,-1,-\left\|v_{1}\right\|^{2}\left\|w_{2}\right\|^{2}\left\|w_{3}\right\|^{2},-\left\|v_{2}\right\|^{2}\left\|w_{1}\right\|^{2}\left\|w_{3}\right\|^{2}\right) \\
& \times \operatorname{msgn}\left(\left\|v_{1}\right\|^{2}\left\|w_{2}\right\|^{2}\left\|w_{3}\right\|^{2},\left\|v_{2}\right\|^{2}\left\|w_{1}\right\|^{2}\left\|w_{3}\right\|^{2}\right) \\
= & \operatorname{msgn}\left(-1,-1,-\left\|v_{1}\right\|^{2}\left\|w_{2}\right\|^{2}\left\|w_{3}\right\|^{2},-\left\|v_{2}\right\|^{2}\left\|w_{1}\right\|^{2}\left\|w_{3}\right\|^{2},\left\|v_{1}\right\|^{2}\left\|w_{2}\right\|^{2}\left\|w_{3}\right\|^{2},\right. \\
& \left.\left\|v_{2}\right\|^{2}\left\|w_{1}\right\|^{2}\left\|w_{3}\right\|^{2}\right) \times \operatorname{msgn}\left(\left\|v_{1}\right\|^{2}\left\|v_{2}\right\|^{2}\left\|w_{1}\right\|^{2}\left\|w_{2}\right\|^{2},\left\|v_{1}\right\|^{2}\left\|v_{2}\right\|^{2}\left\|w_{1}\right\|^{2}\left\|w_{2}\right\|^{2}\right) \\
= & \operatorname{sgn}\left(\left\|v_{1}\right\|^{2}\left\|v_{2}\right\|^{2}\left\|w_{1}\right\|^{2}\left\|w_{2}\right\|^{2}\right),
\end{aligned}
$$

where we used Proposition 4.4 b), c), and d).

As is shown, we deduce the dual cosine law on the hyperbolic sphere $\mathbb{S}_{H}^{2}$

In order to get the dual cosine law on the spherical sphere $\mathbb{S}_{S}^{2}$, we need only $i \cdot d_{S}=d_{H}$ as before.

We considered only triangle with non-lightlike vertex vectors, i.e., without ideal vertices. If we permit lightlike vector, then the values $\|\cdot\|$ become 0 and angles and lengths can be 0 or $\infty$. Even in this degenerated case, we can easily convince the cosine and dual cosine law, too. Therefore we can summarize the cosine law and dual cosine law for $\mathbb{S}_{H}^{2}$ and $\mathbb{S}_{S}^{2}$ in the following theorem.

Theorem 4.13. Letting $A, B, C$ stand for the angles and $a, b, c$ for the extended hyperbolic lengths of opposite sides of a given triangle, we obtain the hyperbolic cosine law and the dual cosine law on the hyperbolic sphere $\mathbb{S}_{H}^{2}$,

$$
\begin{aligned}
\cos C & =\frac{\cosh a \cosh b-\cosh c}{\sinh a \sinh b}, \\
\cosh c & =\frac{\cos A \cos B+\cos C}{\sin A \sin B} .
\end{aligned}
$$


Also we have the spherical cosine law and dual cosine law on the spherical sphere $\mathbb{S}_{S}^{2}$, where $a, b, c$ represent the extended spherical lengths,

$$
\begin{aligned}
\cos C & =\frac{\cos c-\cos a \cos b}{\sin a \sin b}, \\
\cos c & =\frac{\cos A \cos B+\cos C}{\sin A \sin B} .
\end{aligned}
$$

Now we consider the cosine laws on the hyperbolic sphere $\mathbb{S}_{H}^{3}$ or the spherical sphere $\mathbb{S}_{S}^{3}$, then we should consider two more cases of triangles.

If a hyperplane containing the triangle does not intersect to $\partial \mathbb{H}^{3}$, then we can send this triangle to the equator $\left(=\mathbb{S}_{H}^{3} \cap\left\{x \mid x_{0}=0\right\}\right)$ of $\mathbb{S}_{H}^{3}$ by an isometry. Hence the distance becomes $i$ times the distance on the standard Euclidean unit sphere. Therefore the above result of Theorem 4.13 also satisfied by the well known spherical trigonometry.
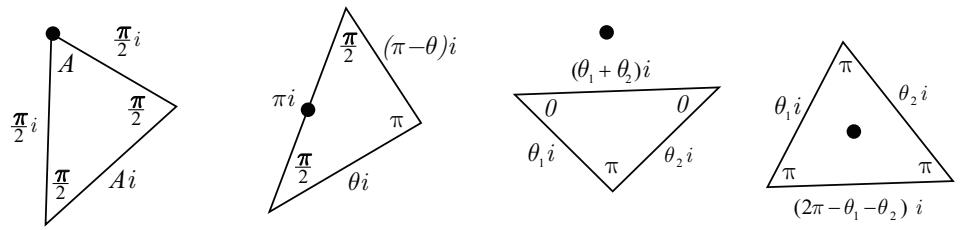

Fig. 11

If a hyperplane containing the triangle is tangent to $\partial \mathbb{H}^{3}$, then there are only four types of triangles in the sense of the intersection of three hemispheres (see Fig. 11).

These four types also satisfy the above cosine laws in some sense, if we permit $\frac{0}{0}=$ a certain number or move the denominator of the formulas to the other side.

Therefore we can conclude a theorem about all kinds of triangles in $\mathbb{S}_{H}^{n}$ or $\mathbb{S}_{S}^{n}$.

Theorem 4.14. For a given triangle in $\mathbb{S}_{H}^{n}$ (resp. $\left.\mathbb{S}_{S}^{n}\right)$, the triangle satisfies the hyperbolic (resp. spherical) cosine and dual cosine laws as in Theorem 4.13.

\subsection{Sine law}

The hyperbolic sine law is easily obtained by the following steps. First we assume that all vertices of a triangle are not ideal vertices. From the dual cosine law for a right triangle with $C=\frac{\pi}{2}$, we have

$$
\cosh b=\frac{\cos A \cos C+\cos B}{\sin A \sin C}=\frac{\cos B}{\sin A},
$$

and also cosine law induces $\cosh c=\cosh a \cosh b$ and

$$
\cos B=\frac{\cosh a \cosh c-\cosh b}{\sinh a \sinh c} .
$$


By substituting (11) and $\cosh c=\cosh a \cosh b$ into (12), we get

$$
\sinh a=\sin A \sinh c .
$$

Now given any triangle with sides $\tilde{a}, \tilde{b}, \tilde{c}$ and angles $\tilde{A}, \tilde{B}, \tilde{C}$, the altitude $h$ corresponding to side $a$, so we can induce $\sinh h=\sin \tilde{A} \sinh \tilde{b}$ and also $\sinh h=\sin \tilde{B} \sinh \tilde{a}$. Here altitude line can be constructed by joining one vertex point and the dual point of the line which passes the other two points. In the proof, the non-ideal vertex condition is necessary used for cancelation. When we consider ideal vertex case, then the sine law also satisfied by easy checking. Therefore we proved the following theorem for hyperbolic sine law and spherical sine law.

Theorem 4.15. Letting $A, B, C$ stand for the angles and $a, b, c$ for the extended hyperbolic lengths of opposite sides of a given triangle, we obtain the hyperbolic sine law on the hyperbolic sphere $\mathbb{S}_{H}^{2}$,

$$
\frac{\sinh a}{\sin A}=\frac{\sinh b}{\sin B}=\frac{\sinh c}{\sin C} .
$$

Also we have the spherical sine law on the spherical sphere $\mathbb{S}_{S}^{2}$, where $a, b, c$ represent the extended spherical lengths,

$$
\frac{\sin a}{\sin A}=\frac{\sin b}{\sin B}=\frac{\sin c}{\sin C} .
$$

We introduce another proof.

Proof. We know

$$
\frac{\sinh ^{2} a}{\sin ^{2} A}=\frac{\sinh ^{2} a \sinh ^{2} b \sinh ^{2} c}{1-\cosh ^{2} a-\cosh ^{2} b-\cosh ^{2} c+2 \cosh a \cosh b \cosh c},
$$

so we conclude $\frac{\sinh ^{2} a}{\sin ^{2} A}=\frac{\sinh ^{2} b}{\sin ^{2} B}=\frac{\sinh ^{2} c}{\sin ^{2} C}$, in particular, it is also satisfied when a denominator or numerator of the formula takes 0 or $\infty$. Now we have to show that $\frac{\sinh a}{\sin A}=\frac{\sinh b}{\sin B}=\frac{\sinh c}{\sin C}$ for a non-ideal vertices triangle.

From $\sinh ^{2} a \sin ^{2} B=\sinh ^{2} b \sin ^{2} A$, it follows continuously that

$$
\sqrt{\sinh ^{2} a \sin ^{2} B}=\sqrt{\sinh ^{2} b \sin ^{2} A},
$$

$\operatorname{msgn}\left(\sinh ^{2} a, \sin ^{2} B\right) \sqrt{\sinh ^{2} a} \sqrt{\sin ^{2} B}=\operatorname{msgn}\left(\sinh ^{2} b, \sin ^{2} A\right) \sqrt{\sinh ^{2} b} \sqrt{\sin ^{2} A}$,

$$
\begin{aligned}
& \operatorname{msgn}\left(\sinh ^{2} a, \sin ^{2} B\right) \operatorname{sgn}(\sinh a) \operatorname{sgn}(\sin B) \sinh a \sin B \\
= & \operatorname{msgn}\left(\sinh ^{2} b, \sin ^{2} A\right) \operatorname{sgn}(\sinh b) \operatorname{sgn}(\sin A) \sinh b \sin A .
\end{aligned}
$$

Hence if

$$
\begin{aligned}
& \operatorname{msgn}\left(\sinh ^{2} a, \sin ^{2} B\right) \operatorname{sgn}(\sinh a) \operatorname{sgn}(\sin B) \\
= & \operatorname{msgn}\left(\sinh ^{2} b, \sin ^{2} A\right) \operatorname{sgn}(\sinh b) \operatorname{sgn}(\sin A)
\end{aligned}
$$

is satisfied, then the proof ends. 
We already know that

$$
\begin{aligned}
\operatorname{sgn}(\sinh a) & =-\operatorname{msgn}\left(-1,-\left\|v_{2}\right\|^{2},-\left\|v_{3}\right\|^{2},-\left\|w_{1}\right\|^{2}\right), \\
\operatorname{sgn}\left(\sinh ^{2} a\right) & =\operatorname{sgn}\left(\left\|v_{2}\right\|^{2}\left\|v_{3}\right\|^{2}\left\|w_{1}\right\|^{2}\right), \\
\operatorname{sgn}(\sin A) & =-\operatorname{msgn}\left(-1,-\left\|v_{1}\right\|^{2},-\left\|w_{2}\right\|^{2},-\left\|w_{3}\right\|^{2}\right), \\
\operatorname{sgn}\left(\sin ^{2} A\right) & =\operatorname{sgn}\left(-\left\|v_{1}\right\|^{2}\left\|w_{2}\right\|^{2}\left\|w_{3}\right\|^{2}\right) .
\end{aligned}
$$

It suffices to show that the following formula is true.

$$
\begin{aligned}
& \operatorname{msgn}\left(-1,-\left\|v_{1}\right\|^{2},-\left\|w_{2}\right\|^{2},-\left\|w_{3}\right\|^{2}\right) \operatorname{msgn}\left(-1,-\left\|v_{1}\right\|^{2},-\left\|v_{3}\right\|^{2},-\left\|w_{2}\right\|^{2}\right) \\
\times & \operatorname{msgn}\left(-1,-\left\|v_{2}\right\|^{2},-\left\|w_{1}\right\|^{2},-\left\|w_{3}\right\|^{2}\right) \operatorname{msgn}\left(-1,-\left\|v_{2}\right\|^{2},-\left\|v_{3}\right\|^{2},-\left\|w_{1}\right\|^{2}\right) \\
\times & \operatorname{msgn}\left(-\left\|v_{1}\right\|^{2}\left\|w_{2}\right\|^{2}\left\|w_{3}\right\|^{2},\left\|v_{1}\right\|^{2}\left\|v_{3}\right\|^{2}\left\|w_{2}\right\|^{2}\right) \\
\times & \operatorname{msgn}\left(-\left\|v_{2}\right\|^{2}\left\|w_{1}\right\|^{2}\left\|w_{3}\right\|^{2},\left\|v_{2}\right\|^{2}\left\|v_{3}\right\|^{2}\left\|w_{1}\right\|^{2}\right)=1 .
\end{aligned}
$$

We left the proof of the msgn equality as an easy exercise for readers.

As is shown in Theorem 4.14, we also similarly induce the sine law on $\mathbb{S}_{H}^{n}$ or $\mathbb{S}_{S}^{n}$ (easy check). Therefore we can conclude the following theorem.

Theorem 4.16. For a given triangle in $\mathbb{S}_{H}^{n}$ (resp. $\left.\mathbb{S}_{S}^{n}\right)$, the triangle satisfies the hyperbolic (resp. spherical) sine law as in Theorem 4.15.

\subsection{Applications for hyperbolic polygons}
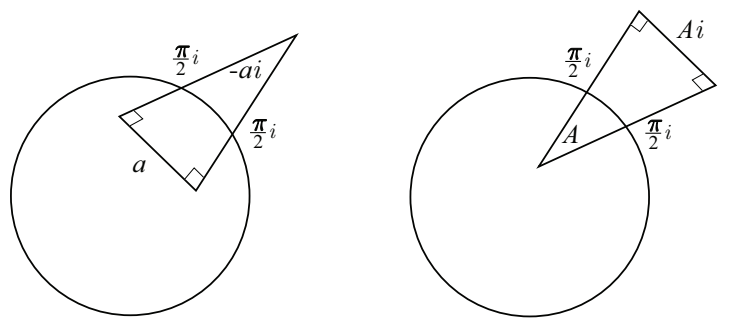

Fig . 12

There are many formulas for Lambert quadrilaterals and pentagons and hexagons, and these were shown by an unified method which starts from a rectangular hexagon in Fenchel's book [8]. Also the above general version of cosine laws and sine law also induce those formulas about all polygons which was mentioned in [8]. Readers can notice that our interpretation gives an easy and natural way to understand.

Here we only need the definition of angle and the fact that the distance between $x$ and $x^{\perp}$ is $\frac{\pi}{2} i$ (see Fig. 12). Also it is convenient to remember that $\sin i x=i \sinh x, \sinh i x=i \sin x, \sinh (x+\pi i)=-\sinh x, \sinh \left(x+\frac{\pi}{2} i\right)=i \cosh x$, 
$\cos i x=\cosh x, \cosh i x=\cos x, \cosh (x+\pi i)=-\cosh x, \cosh \left(x+\frac{\pi}{2} i\right)=i \sinh x$.

Now we examine four special cases. At first consider a quadrilateral with consecutive two right angles shown in Fig. 13 below. We know that the lengths between 1,2 and 2,3 and 3,1 are $a+\frac{\pi}{2} i, c$ and $b+\frac{\pi}{2} i$ respectively, and the angles at 1,2 and 3 are $-d i, B$ and $A$.

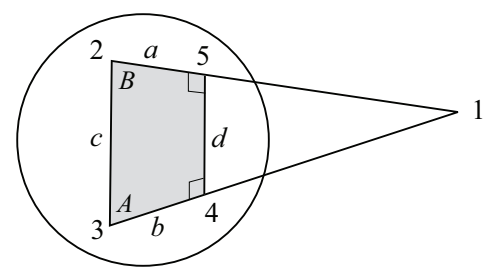

Fig. 13

From the cosine law for a triangle $(1,2,3)$, we obtain formulas:

$$
\begin{aligned}
\cos (-d i) & =\frac{\cosh \left(a+\frac{\pi}{2} i\right) \cosh \left(b+\frac{\pi}{2} i\right)-\cosh c}{\sinh \left(a+\frac{\pi}{2} i\right) \sinh \left(b+\frac{\pi}{2} i\right)} \rightarrow \cosh d=\frac{\sinh a \sinh b+\cosh c}{\cosh a \cosh b}, \\
\cos A & =\frac{\cosh c \cosh \left(b+\frac{\pi}{2} i\right)-\cosh \left(a+\frac{\pi}{2} i\right)}{\sinh c \sinh \left(b+\frac{\pi}{2} i\right)} \rightarrow \cos A=\frac{\cosh c \sinh b-\sinh a}{\sinh c \cosh b} .
\end{aligned}
$$

Also the dual cosine law induces

$$
\begin{aligned}
\cosh \left(a+\frac{\pi}{2} i\right) & =\frac{\cos B \cos (-d i)+\cos A}{\sin B \sin (-d i)} \rightarrow \sinh a=\frac{\cos B \cosh d+\cos A}{\sin B \sinh d}, \\
\cosh c & =\frac{\cos A \cos B+\cos (-d i)}{\sin A \sin B} \rightarrow \cosh c=\frac{\cos A \cos B+\cosh d}{\sin A \sin B} .
\end{aligned}
$$

And the sine law implies

$$
\frac{\sinh \left(a+\frac{\pi}{2} i\right)}{\sin A}=\frac{\sinh \left(b+\frac{\pi}{2} i\right)}{\sin B}=\frac{\sinh c}{\sin (-d i)} \rightarrow \frac{\cosh a}{\sin A}=\frac{\cosh b}{\sin B}=\frac{\sinh c}{\sinh d} .
$$

A rectangular hyperbolic hexagon can be perceived as a truncated triangle (see Fig. 14). So the triangle has lengths $a+\pi i, b+\pi i$ and $c+\pi i$, and angles $-A i,-B i$ and $-C i$.

From the cosine law and dual cosine law, we get

and

$$
\cosh C=\frac{\cosh a \cosh b+\cosh c}{\sinh a \sinh b}
$$

$$
\cosh c=\frac{\cosh A \cosh B+\cosh C}{\sinh A \sinh B} .
$$




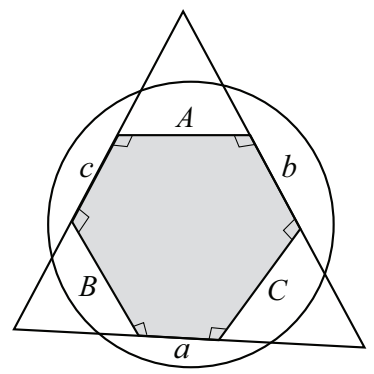

Fig . 14

The sine law shows

$$
\frac{\sinh a}{\sinh A}=\frac{\sinh b}{\sinh B}=\frac{\sinh c}{\sinh C} .
$$

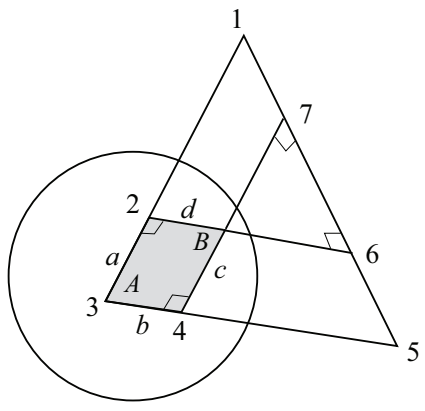

Fig . 15

A hyperbolic quadrilateral with two opposite rectangular angles also can be applicable (see Fig. 15). We know that

$$
\overline{13}=a+\frac{\pi}{2} i, \quad \overline{35}=b+\frac{\pi}{2} i, \quad \overline{15}=\overline{16}+\overline{57}-\overline{67}=\frac{\pi}{2} i+\frac{\pi}{2} i-B i,
$$

and

$$
\angle 1=-i \cdot \overline{26}=-i\left(d+\frac{\pi}{2} i\right)=\frac{\pi}{2}-d i, \quad \angle 5=\frac{\pi}{2}-c i, \quad \angle 3=A .
$$

Hence by generalized hyperbolic cosine and sine laws, we get

$$
\begin{gathered}
\cos A=\frac{\sinh a \sinh b-\cos B}{\cosh a \cosh b} . \\
\sinh a=\frac{\cos A \sinh d+\sinh c}{\sin A \cosh d}, \\
\frac{\sin B}{\sin A}=\frac{\cosh a}{\cosh c}=\frac{\cosh b}{\cosh d} .
\end{gathered}
$$




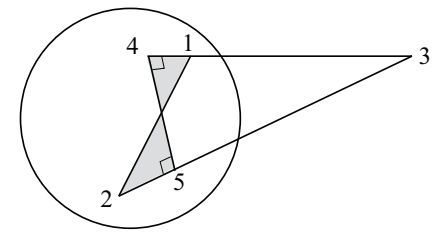

Fig . 16

Even in a self intersecting quadrilateral (see Fig. 16), we can apply the generalized hyperbolic trigonometry. From the general triangle $\triangle(1,2,3)$, we will get the trigonometry of the quadrilateral $(1,2,5,4)$.

One can easily examine the other formulas for various hyperbolic polygons in the similar way.

\subsection{Applications for de Sitter polygons}
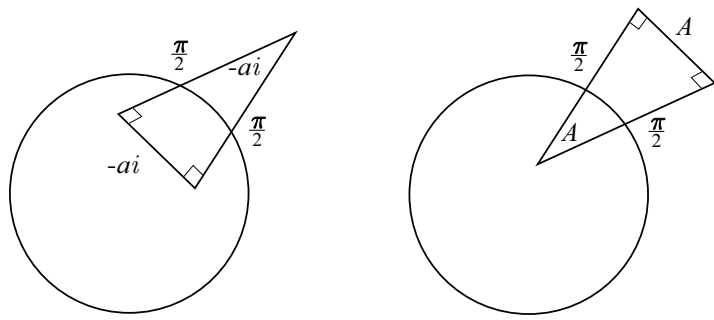

Fig . 17

The generalized spherical cosine and sine laws can be used for the polygons on $S_{1}^{n}$. So we can get many formulas for polygons on $S_{1}^{n}$ by the similar way of $\S 4.3$, those formulas are not unknown yet as I know. Especially for a triangle contained in $S_{1}^{n}$, Dzan [7] also induced the same spherical type cosine and sine laws.

First, we need the basic facts about lengths and angles: The distance between $x$ and $x^{\perp}$ is $\frac{\pi}{2}$, and the angle $\angle\left(x_{p}, y_{p}\right)$ is $d_{S}(x, y)$ (see Fig. 17). We have to define a timelike (resp. spacelike) edge as the geodesic edge whose tangent vector is timelike (resp. spacelike) vector, then we know that a time edge inside of the Lorentzian part has positive pure imaginary length and a space edge has positive real length on the extended de Sitter space (see Convention 2.4).

Now we examine the two cases. First one is a Lambert quadrilateral shown in Fig. 18. We know that the lengths between 1,2 and 1,3 and 2,3 are $a i+\frac{\pi}{2}$ and $c i+\frac{\pi}{2}$ and $d$ respectively, and the angles at 1,2 and 3 are $b, \frac{\pi}{2}$ and $\phi$. Here $\phi$ is a complex number and all the others are positive real numbers. From the 


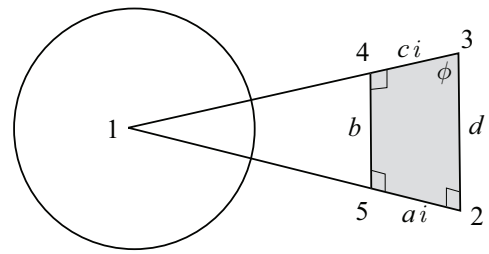

Fig . 18

spherical cosine law for a right triangle $(1,2,3)$, we obtain three formulas

$$
\begin{aligned}
\cos b & =\frac{\sinh a \sinh c+\cos d}{\cosh a \cosh c}, \\
\cos \phi & =-i \frac{\sinh a-\sinh c \cos d}{\cosh c \sin d}, \\
\sinh c & =\cos d \sinh a .
\end{aligned}
$$

Also the spherical dual cosine law induces

$$
\cos b=\cos d \sin \phi, \cos \phi=-i \sinh a \sin b, \text { and } \sinh a=i \cot b \cot \phi .
$$

The formulas (13) and the middle one of (14) induce the inequality $\sinh a>$ $\sinh c \cos d$. And the sine law gives us

$$
\frac{\sin d}{\sin b}=\cosh c=\frac{\cosh a}{\sin \phi} \text {. }
$$

A pentagon with four right angles in the de Sitter space can be perceived as a truncated triangle (see Fig. 19). From the figure, the triangle $(1,2,3)$ has three side of lengths $a i+\frac{\pi}{2}, e i+\frac{\pi}{2}$ and $c i+\pi$ and three angles $\phi, b$ and $d$. Here $\phi$ is a complex number and all the others are positive real numbers. So we get six formulas from the spherical cosine and dual cosine laws.

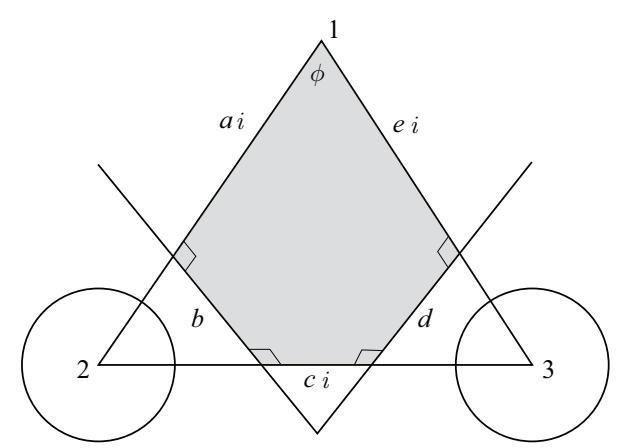

Fig . 19 
They are

$$
\begin{aligned}
\cos b & =\frac{\sinh a \cosh c+\sinh e}{\cosh a \sinh c}, \\
\cos d & =\frac{\sinh e \cosh c+\sinh a}{\cosh e \sinh c}, \\
\cos \phi & =\frac{\sinh a \sinh e-\cosh c}{\cosh a \cosh e},
\end{aligned}
$$

and

$$
\begin{aligned}
-\cosh c & =\frac{\cos b \cos d+\cos \phi}{\sin b \sin d}, \\
-i \sinh a & =\frac{\cos b \cos \phi+\cos d}{\sin b \sin \phi}, \\
-i \sinh e & =\frac{\cos d \cos \phi+\cos b}{\sin d \sin \phi} .
\end{aligned}
$$

We can easily show that the angle $b$ and $d$ are smaller than $\frac{\pi}{2}$, so we get $\cos b, \cos d>0$. Then from the first formula of (16), we have $\cos \phi<0$, so the third formula of (15) gives us an inequality, $\sinh a \sinh e<\cosh c$. And the sine law implies

$$
-i \frac{\sinh c}{\sin \phi}=\frac{\cosh e}{\sin b}=\frac{\cosh a}{\sin d} .
$$

Readers can easily induce the trigonometry formula for some de Sitter polygons with six variables of lengths and angles and the others rectangular angles by the similar way.

\subsection{Three dimensional application}

Let's consider the following well-known tangent law (see [9]) for the hyperbolic orthoscheme as a three dimensional application for Theorems 4.13 and 4.15 .

$$
\tanh a \tan \alpha=\tanh b \cot \beta=\tanh c \tan \gamma=\frac{\sqrt{\cos ^{2} \beta-\sin ^{2} \alpha \sin ^{2} \gamma}}{\cos \alpha \cos \gamma},
$$

where $\alpha, \beta, \gamma$ denote the dihedral angles and all the other dihedral angles are $\frac{\pi}{2}$, and $\overline{12}=a, \overline{14}=b, \overline{34}=c$ (see Fig. 20).

A general position orthoscheme in the extended hyperbolic space has triangle faces lying on the extended space and dual triangles which are obtained by the intersection of the extensions of three faces and the dual plane at the common point of the three faces. Then four facial triangles and four dual triangles are lying on the extended hyperbolic space, so we can apply Theorem 4.13 and 4.15 , i.e., extended hyperbolic trigonometry. From the general trigonometry on the general position orthoscheme, we easily induce the following relations:

(18) $\tanh ^{2} a \tan ^{2} \alpha=\tanh ^{2} b \cot ^{2} \beta=\tanh ^{2} c \tan ^{2} \gamma=\frac{\cos ^{2} \beta-\sin ^{2} \alpha \sin ^{2} \gamma}{\cos ^{2} \alpha \cos ^{2} \gamma}$. 


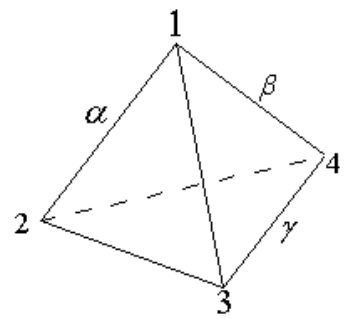

Fig . 20

We need one more process from the formula (18) to the formula (17). Maybe there exists a msgn technique proof (see $\S 4.1,4.2)$ at the last step, however we prove the step by some elementary method for each case. For a usual hyperbolic orthoscheme, the positive area condition of triangles $(1,2,3)$ and $(2,3,4)$ induce $0<\alpha, \gamma<\frac{\pi}{2}$, and the area of $(2,3,4)$ and the trigonometry at dual triangle at the vertex 4 induce $0<\beta<\frac{\pi}{2}$.

The acute angle property of $\alpha, \beta, \gamma$ and gram matrix property $\cos ^{2} \beta-$ $\sin ^{2} \alpha \sin ^{2} \gamma>0$ for a hyperbolic orthoscheme trivially induce the formula (17) from the formula (18).

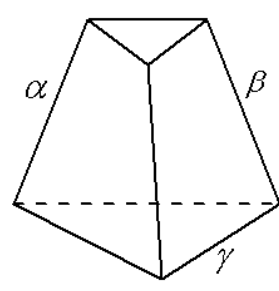

(i)

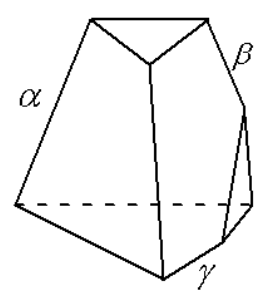

(ii)

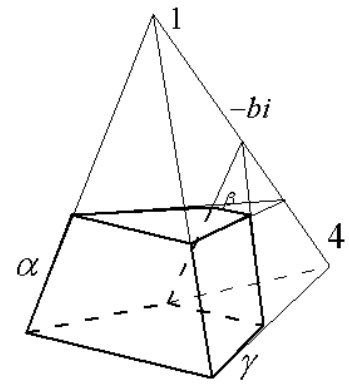

(iii)

Fig . 21

If the vertex 1 of a hyperbolic orthoscheme (see Fig. 20) lies on the Lorentzian part, then we can cut the orthoscheme by a dual plane $1^{\perp}$ and obtain a prism, i.e., hyperbolic one vertex truncated orthoscheme (see Fig. 21 (i)).

Additionally one more truncation by a dual plane $4^{\perp}$ in Fig. 20, then we obtain a hyperbolic two vertices truncated orthoscheme (see Fig. 21 (ii)). If the edge 14 in Fig. 20 is located in the Lorentzian part, we can make a hyperbolic Lambert cube (see Fig. 21 (iii)). Then the corresponding lengths at 
the dihedral angles $\alpha, \beta, \gamma$ for three polyhedra in Fig. 21 are denoted by $a, b, c$, respectively.

In order to find the corresponding tangent laws for specific cases, we have to substitute $\alpha, \beta, \gamma, a, b, c$ at the general formula (18) to suitable values.

The facial polygons in (i), (ii), and (iii) cases easily induce that all dihedral angles $\alpha, \beta, \gamma$ are acute, so the following tangent laws are immediately induced from the formula (18).

Case (i), by substitution $a \rightarrow a+\frac{\pi}{2} i, b \rightarrow b+\frac{\pi}{2} i$ in (17), we obtain

$$
\operatorname{coth} a \tan \alpha=\operatorname{coth} b \cot \beta=\tanh c \tan \gamma=\frac{\sqrt{\cos ^{2} \beta-\sin ^{2} \alpha \sin ^{2} \gamma}}{\cos \alpha \cos \gamma} .
$$

Case (ii), by substitution $a \rightarrow a+\frac{\pi}{2} i, b \rightarrow b+\pi i, c \rightarrow c+\frac{\pi}{2} i$ in (17), we obtain

$$
\operatorname{coth} a \tan \alpha=\tanh b \cot \beta=\operatorname{coth} c \tan \gamma=\frac{\sqrt{\cos ^{2} \beta-\sin ^{2} \alpha \sin ^{2} \gamma}}{\cos \alpha \cos \gamma} .
$$

Case (iii), by substitution $a \rightarrow a+\frac{\pi}{2} i, b \rightarrow i(\pi-\beta), c \rightarrow c+\frac{\pi}{2} i, \beta \rightarrow-b i$ in (17), we obtain

$$
\operatorname{coth} a \tan \alpha=\operatorname{coth} b \tan \beta=\operatorname{coth} c \tan \gamma=\frac{\sqrt{\cosh ^{2} b-\sin ^{2} \alpha \sin ^{2} \gamma}}{\cos \alpha \cos \gamma} .
$$

Considering of the extended de Sitter space instead of the extended hyperbolic space, immediately we get the tangent laws for spherical orthoscheme and spherical one vertex truncated orthoscheme and spherical Lambert cube by substitution $a, b, c \rightarrow i a, i b, i c$, respectively:

Case (iv), spherical orthoscheme

$$
\tan a \tan \alpha=\tan b \cot \beta=\tan c \tan \gamma=\frac{\sqrt{\sin ^{2} \alpha \sin ^{2} \gamma-\cos ^{2} \beta}}{\cos \alpha \cos \gamma} .
$$

Case (v), spherical one vertex truncated orthoscheme

$$
-\cot a \tan \alpha=-\cot b \cot \beta=\tan c \tan \gamma=\frac{\sqrt{\sin ^{2} \alpha \sin ^{2} \gamma-\cos ^{2} \beta}}{\cos \alpha \cos \gamma} .
$$

Case (vi), spherical Lambert cube

(21) $-\cot a \tan \alpha=-\cot b \tan \beta=-\cot c \tan \gamma=\frac{\sqrt{\sin ^{2} \alpha \sin ^{2} \gamma-\cos ^{2} b}}{\cos \alpha \cos \gamma}$.

The formula (19) was introduced by Vinberg [1], and the formula (21) by Derevnin and Mednykh [4], and four tangent laws in hyperbolic space by Kellerhals [9].

Because of the non-existence of a right-angled spherical pentagon, the spherical two vertices truncated orthoscheme does not exist. As we mentioned hyperbolic cases, the square of the three formulas (19), (20), and (21) are trivially satisfied. To complete the proofs, we need final geometric steps such as from (18) to (17). 
The step from $(20)^{2}$ to $(20)$ is satisfied by the following condition. The trigonometry of the facial polygons on spherical one vertex truncated orthoscheme easily induces $0<a, b, c, \alpha, \beta<\frac{\pi}{2}<\gamma<\pi$, hence the final step $(20)^{2}$ to (20) is completed. Also the property on Lambert cube $0<a, b, c<\frac{\pi}{2}<$ $\alpha, \beta, \gamma<\pi$ is easily induced and implies the step $(21)^{2}$ to $(21)$.

To gain the formula (19), we should show that $\tan a \tan \alpha, \tan b \cot \beta, \tan c$ $\tan \gamma, \cos \alpha \cos \gamma$ have the same \pm sign, i.e.,

$$
\tan a \tan \alpha \sim \tan b \cot \beta \sim \tan c \tan \gamma \sim \cos \alpha \cos \gamma
$$

The spherical trigonometry of triangles $(1,2,3)$ and $(2,3,4)$ in Fig. 20 implies $\cos a \sim \cos \gamma$ and $\cos c \sim \cos \alpha$, and the triangle $(1,3,4)$ and the dual spherical triangle at 4 induce $\cos \theta=\cot \beta \cot \gamma, \cos x=\frac{\cos \theta}{\sin \delta}, \cos b=\cos x \cos c$ (see Fig. $22)$.
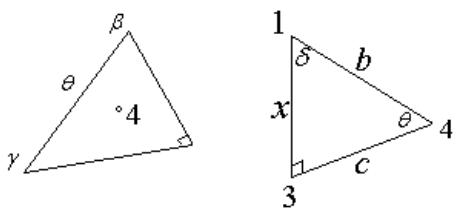

Fig . 22

So we have that

$$
\cos b=\cos x \cos c \sim \cos \theta \cos c \sim \cot \beta \cot \gamma \cos \alpha \sim \cos \alpha \cos \beta \cos \gamma
$$

and $\cos b \cos \beta \sim \cos \alpha \cos \beta \cos \gamma \cos \beta \sim \cos \alpha \cos \gamma$. Hence the same sign property is proved. In order to prove the formula (19) for the $\frac{\pi}{2}$ dihedral angles or lengths cases, we only need the assumption of natural infinite calculation such as $\infty \cdot \infty=\infty$, then we can check the formula (19) for those special cases. Therefore we can conclude the formula (19).

We proved the tangent laws for specific 7 cases in the unified way. Note that the tangent law is important to calculate the volume. Additionally, we show other laws which was mentioned in [4] or some papers of Mednykh's school to the seven cases. They showed sine-cosine laws in their papers.

Now we can explain the sine-cosine laws in the extended hyperbolic space point of view as well. To obtain a unified proof, we only follow the same method given in the tangent law cases. Particularly, if we know one law among the following 21 laws, we can automatically induce other 20 laws by the substitution rule and the symmetry of Lambert cube.

- Hyperbolic orthoscheme

$$
\frac{\cos \beta}{\cosh b}=\frac{\sin \alpha \sin \gamma}{\cosh a \cosh c}, \frac{\sin \beta}{\sinh b}=\frac{\cos \alpha \sin \gamma}{\sinh a \cosh c}, \frac{\sin \beta}{\sinh b}=\frac{\sin \alpha \cos \gamma}{\cosh a \sinh c} \text {. }
$$


- Hyperbolic one vertex truncated orthoscheme

$\frac{\cos \beta}{\sinh b}=\frac{\sin \alpha \sin \gamma}{\sinh a \cosh c}, \frac{\sin \beta}{\cosh b}=\frac{\cos \alpha \sin \gamma}{\cosh a \cosh c}, \frac{\sin \beta}{\cosh b}=\frac{\sin \alpha \cos \gamma}{\sinh a \sinh c}$.

- Hyperbolic two vertices truncated orthoscheme

$\frac{\cos \beta}{\cosh b}=\frac{\sin \alpha \sin \gamma}{\sinh a \sinh c}, \frac{\sin \beta}{\sinh b}=\frac{\cos \alpha \sin \gamma}{\cosh a \sinh c}, \frac{\sin \beta}{\sinh b}=\frac{\sin \alpha \cos \gamma}{\sinh a \cosh c}$.

- Hyperbolic Lambert cube

$\frac{\sin \alpha}{\sinh a} \frac{\cos \beta}{\cosh b} \frac{\sin \gamma}{\sinh c}=1, \frac{\cos \alpha}{\cosh a} \frac{\sin \beta}{\sinh b} \frac{\sin \gamma}{\sinh c}=1, \frac{\sin \alpha}{\sinh a} \frac{\sin \beta}{\sinh b} \frac{\cos \gamma}{\cosh c}=1$.

- Spherical orthoscheme

$$
\frac{\cos \beta}{\cos b}=\frac{\sin \alpha \sin \gamma}{\cos a \cos c}, \frac{\sin \beta}{\sin b}=\frac{\cos \alpha \sin \gamma}{\sin a \cos c}, \frac{\sin \beta}{\sin b}=\frac{\sin \alpha \cos \gamma}{\cos a \sin c} .
$$

- Spherical one vertex truncated orthoscheme

$$
\frac{\cos \beta}{\sin b}=\frac{\sin \alpha \sin \gamma}{\sin a \cos c}, \frac{\sin \beta}{\cos b}=\frac{\cos \alpha \sin \gamma}{\cos a \cos c}, \frac{\sin \beta}{\cos b}=-\frac{\sin \alpha \cos \gamma}{\sin a \sin c}
$$

- Spherical Lambert cube

$$
\frac{\sin \alpha}{\sin a} \frac{\cos \beta}{\cos b} \frac{\sin \gamma}{\sin c}=-1, \frac{\cos \alpha}{\cos a} \frac{\sin \beta}{\sin b} \frac{\sin \gamma}{\sin c}=-1, \frac{\sin \alpha}{\sin a} \frac{\sin \beta}{\sin b} \frac{\cos \gamma}{\cos c}=-1 \text {. }
$$

Lastly we want to remark some problems. Even though the properties on the extended space are very natural, our proof for the trigonometry is, more or less, artificial. Hence we leave the following problem.

Problem 1. Find a natural proof for the trigonometry on the extended hyperbolic space or the extended de Sitter space.

We can consider a triangle area formula on the extended hyperbolic space and the extended de Sitter space. Particularly, an area formula for a triangle with angles $A, B, C$ is represented by $\pi-A-B-C$ on $\mathbb{S}_{H}^{n}$ (naturally $A+B+C-\pi$ on $\left.\mathbb{S}_{S}^{n}\right)($ see $[2])$.

If we apply the cosine law on the extended hyperbolic space to the area formula $S=\pi-A-B-C$, then we obtain another area formula $S_{1}(a, b, c)$ with three edge length variables $a, b, c$,

$$
\begin{aligned}
S_{1}=\pi & -\cos ^{-1}\left(\frac{\cosh b \cosh c-\cosh a}{\sinh b \sinh c}\right)-\cos ^{-1}\left(\frac{\cosh a \cosh c-\cosh b}{\sinh a \sinh c}\right) \\
& -\cos ^{-1}\left(\frac{\cosh a \cosh b-\cosh c}{\sinh a \sinh b}\right) .
\end{aligned}
$$

We already know another area formula $S_{2}(a, b, c)$ (see [1], there is a misprint that is easily checked by considering $a=b=c=\infty$ ) for a triangle on the hyperbolic space,

$$
\tan ^{2} \frac{S_{2}}{4}=\tanh \frac{p}{2} \tanh \frac{p-a}{2} \tanh \frac{p-b}{2} \tanh \frac{p-c}{2}, \quad \text { where } p=\frac{a+b+c}{2} .
$$


Two functions $S_{1}$ and $S_{2}$ are complex multi-valued functions on $\mathbb{C}^{3}$, and coincide each other when the triangle lies on the hyperbolic space, i.e., have the same value on a domain $U \subset \mathbb{R}^{3} \subset \mathbb{C}^{3}$. Hence $S_{1}$ and $S_{2}$ coincide each other on $\mathbb{C}^{3}$ with the same branch cuts, and so they have the same value for a triangle on the extended hyperbolic space.

We know the principle $i\|v\|_{S}=\|v\|_{H}$, which comes from Conventions 2.2 and 2.4, for two norms $\|v\|_{H}$ and $\|v\|_{S}$ of any directed tangent vector $v$, where $\|\cdot\|_{H}$ (resp. $\|\cdot\|_{S}$ ) denotes a vector norm on the extended hyperbolic space (resp. extended de Sitter space). So any 2-dimensional volume elements $d V_{H}$ and $d V_{S}$ for a given point on $\mathbb{S}_{H}^{n}$ and $\mathbb{S}_{S}^{n}$, respectively, give a natural relation $i^{2} \cdot d V_{S}=d V_{H}$.

Therefore we get an area formula for a triangle on the extended de Sitter space as well as on the spherical space,

$$
\begin{gathered}
\tan ^{2} \frac{-S_{2}}{4}=\tanh \frac{p i}{2} \tanh \frac{(p-a) i}{2} \tanh \frac{(p-b) i}{2} \tanh \frac{(p-c) i}{2}, \\
\text { i.e., } \tan ^{2} \frac{S_{2}}{4}=\tan \frac{p}{2} \tan \frac{p-a}{2} \tan \frac{p-b}{2} \tan \frac{p-c}{2}, \quad \text { where } p=\frac{a+b+c}{2} .
\end{gathered}
$$

As a result, we can anticipate the following principle by Cho and Kim.

Problem 2. If a single or multivalued analytic formula with geometric quantity variables is satisfied on the hyperbolic space, then we can obtain the corresponding formula on the spherical space by changing of all variables with a principle that $k$-dimensional hyperbolic variable is replaced by $i^{k} \times$ corresponding $k$-dimensional spherical variable, for example, hyperbolic angle $\theta \rightarrow$ spherical angle $\theta$ and hyperbolic length $l \rightarrow i \times$ spherical length $l$ and so on.

In fact, if an $n$-dimensional (the highest dimension among the variables' dimensions) analytic formula is satisfied on the $n$-dimensional hyperbolic space (resp. spherical space) and if we prove that the analytic formula also holds in the $(n+1)$-dimensional extended hyperbolic space (resp. extended de Sitter space), then Problem 2 is automatically satisfied by the comparison of the extended hyperbolic space and the extended de Sitter space.

In the above problem, if we change the contour for the extended space, the value $i^{k}$ can be replaced by $(-i)^{k}, k=1,2,3, \ldots$. So any single-valued analytic formula $f$, which comes from Problem 2, must have a symmetry for $i$ and $-i$, i.e.,

$$
\begin{aligned}
& f\left(\ldots, i^{k} \cdot k \text {-dim var., } \ldots, i^{n} \cdot n \text {-dim var. }\right) \\
= & f\left(\ldots,(-i)^{k} \cdot k \text {-dim var., } \ldots,(-i)^{n} \cdot n \text {-dim var. }\right) .
\end{aligned}
$$

For hyperbolic and spherical triangles, Lobachevsky even knew the principle for the hyperbolic and spherical trigonometry laws. And for $n$-dimensional hyperbolic and spherical simplices, Vinberg [14] clarified the principle on an analytic volume formula represented by their dihedral angles. 


\section{References}

[1] D. V. Alekseevskij, E. B. Vinberg, and A. S. Solodovnikov, Geometry of spaces of constant curvature, Geometry, II, 1-138, Encyclopaedia Math. Sci., 29, Springer, Berlin, 1993.

[2] Y. Cho and H. Kim, The analytic continuation of hyperbolic space, arXiv:math. MG/0612372.

[3] - Volume of $C^{1, \alpha}$-boundary domain in extended hyperbolic space, J. Korean Math. Soc. 43 (2006), no. 6, 1143-1158.

[4] D. Derevnin and A. Mednykh, On the volume of spherical Lambert cube, arXiv:math. MG/0212301.

[5] J. J. Dzan, Gauss-Bonnet formula for general Lorentzian surfaces, Geom. Dedicata 15 (1984), no. 3, 215-231.

[6] Sectorial measure with applications to non-Euclidean trigonometries, Mitt. Math. Ges. Hamburg 13 (1993), 179-197.

[7] _ Trigonometric Laws on Lorentzian sphere $S_{1}^{2}$, J. Geom. 24 (1985), no. 1, 6-13.

[8] W. Fenchel, Elementary Geometry in Hyperbolic Space, Walter de Gruyter, Berlin New York, 1989.

[9] R. Kellerhals, On the volume of hyperbolic polyhedra, Math. Ann. 285 (1989), no. 4, 541-569.

[10] B. O'Neill, Semi-Riemannian Geometry, Academic Press, New York, London Paris, 1983.

[11] J. G. Ratcliffe, Foundations of Hyperbolic Manifolds, Graduate Texts in Mathematics, 149. Springer-Verlag, New York, 1994.

[12] J. M. Schlenker, Métriques sur les polyèdres hyperboliques convexes, J. Differential Geom. 48 (1998), no. 2, 323-405.

[13] W. P. Thurston, Three-Dimensional Geometry and Topology, Princeton University Press, Princeton New Jersey, 1997.

[14] E. B. Vinberg, Volumes of non-Euclidean Polyhedra, Russian Math. Surveys 48 (1993), no. $2,15-45$.

Department of Mathematics

UNIVERSiTy OF SEOUL

SEOUL 130-743, KOREA

E-mail address: yhcho@uos.ac.kr 